\title{
Dynamical Analysis of a Viral Infection Model with Delays in Computer Networks
}

\author{
Zizhen Zhang ${ }^{1,2}$ and Huizhong Yang ${ }^{2}$ \\ ${ }^{1}$ School of Management Science and Engineering, Anhui University of Finance and Economics, Bengbu 233030, China \\ ${ }^{2}$ Key Laboratory of Advanced Process Control for Light Industry (Ministry of Education), Jiangnan University, Wuxi 214122, China
}

Correspondence should be addressed to Huizhong Yang; yhz@jiangnan.edu.cn

Received 22 July 2014; Accepted 9 November 2014

Academic Editor: Baocang Ding

Copyright (c) 2015 Z. Zhang and H. Yang. This is an open access article distributed under the Creative Commons Attribution License, which permits unrestricted use, distribution, and reproduction in any medium, provided the original work is properly cited.

This paper is devoted to the study of an SIRS computer virus propagation model with two delays and multistate antivirus measures. We demonstrate that the system loses its stability and a Hopf bifurcation occurs when the delay passes through the corresponding critical value by choosing the possible combination of the two delays as the bifurcation parameter. Moreover, the direction of the Hopf bifurcation and the stability of the bifurcating periodic solutions are determined by means of the center manifold theorem and the normal form theory. Finally, some numerical simulations are performed to illustrate the obtained results.

\section{Introduction}

With the rapid development of computer technologies and network applications, the threat of computer viruses to the world would become increasingly serious. It is of vital importance to understand how computer viruses spread over computer network and to control the computer viruses' propagation in computer networks. To this end, many mathematical models have been studied to illustrate the dynamical behavior of computer viruses spreading since Murray [1] suggested that computer viruses share some traits of biological viruses. In [2, 3], Kephart and White used the SIS model to describe the propagation of computer viruses. In [4], Zou et al. investigated how the spread of red worms is affected by the worm characteristics based on the SIR model. In $[5,6]$, Yuan et al. proposed the SEIR computer virus model and studied the dynamics of the model, respectively. In [7], Mishra and Pandey formulated an SEIRS model for the transmission of worms in computer network through vertical transmission. In addition, there are also some researchers who proposed the computer virus models with vaccination and quarantine strategy [8-10].

In fact, many computer viruses have different kinds of delays when the viruses spread, such as latent period delay
$[11,12]$, immunity period delay [12-15], and the delay due to the period that the anti-virus software needs to clean the viruses [6]. In [12], Feng et al. proposed the following computer virus propagation model with dual delays and multistate antivirus measures based on the classical SIR epidemic model in [16]:

$$
\begin{aligned}
\frac{d S(t)}{d t}= & p A-\beta S\left(t-\tau_{1}\right) I\left(t-\tau_{1}\right)-(\mu+\gamma) S(t) \\
& +\delta R\left(t-\tau_{2}\right), \\
\frac{d I(t)}{d t}= & \beta S\left(t-\tau_{1}\right) I\left(t-\tau_{1}\right)-(\mu+\alpha) I(t), \\
\frac{d R(t)}{d t}= & (1-p) A+\gamma S(t)+\alpha I(t)-\delta R\left(t-\tau_{2}\right)-\mu R(t),
\end{aligned}
$$

where $S(t), I(t)$, and $R(t)$ represent the numbers of susceptible, infected, and recovered hosts in computer networks at time $t$, respectively. $A$ is the number of the hosts which are attached to the computer networks and $p$ is the proportion of the new hosts which are susceptible. $\mu$ is the death rate of the hosts. $\alpha, \beta, \gamma$, and $\delta$ are the state transition rates between the classes $S, I$, and $R$. $\tau_{1} \geq 0$ is the latent period of the computer 
viruses and $\tau_{2} \geq 0$ is the temporary immune period of the recovered hosts. For the convenience of analysis, Feng et al. [12] let $\tau_{1}=\tau_{2}$; then, system (1) becomes the following form:

$$
\begin{aligned}
\frac{d S(t)}{d t}= & p A-\beta S(t-\tau) I(t-\tau)-(\mu+\gamma) S(t) \\
& +\delta R(t-\tau) \\
\frac{d I(t)}{d t}= & \beta S(t-\tau) I(t-\tau)-(\mu+\alpha) I(t), \\
\frac{d R(t)}{d t}= & (1-p) A+\gamma S(t)+\alpha I(t)-\delta R(t-\tau)-\mu R(t) .
\end{aligned}
$$

By regarding the time delay $\tau$ as the bifurcation parameter, Feng et al. [12] studied the existence and properties of Hopf bifurcation of system (2). As is known, it needs some time to clean the viruses in the infected hosts for the antivirus software. Therefore, it is reasonable to take into account the time delay due to the period that the antivirus software uses to clean the viruses in the infected hosts in system (2). To this end, we consider the following system with two delays:

$$
\begin{aligned}
\frac{d S(t)}{d t}= & p A-\beta S\left(t-\tau_{1}\right) I\left(t-\tau_{1}\right)-(\mu+\gamma) S(t) \\
& +\delta R\left(t-\tau_{1}\right), \\
\frac{d I(t)}{d t}= & \beta S\left(t-\tau_{1}\right) I\left(t-\tau_{1}\right)-\mu I(t)-\alpha I\left(t-\tau_{2}\right), \\
\frac{d R(t)}{d t}= & (1-p) A+\gamma S(t)+\alpha I\left(t-\tau_{2}\right)-\delta R\left(t-\tau_{1}\right) \\
& -\mu R(t),
\end{aligned}
$$

where $\tau_{1} \geq 0$ is the time delay due to the latent period of the computer viruses and the temporary immune period of the recovered hosts. $\tau_{2} \geq 0$ is the time delay due to the period that the antivirus software uses to clean the viruses in the infected hosts.

The remaining materials of this paper are organized in this fashion: local stability and existence of local Hopf bifurcation are discussed in Section 2. Properties of the Hopf bifurcation such as the direction and stability are investigated in Section 3. Some numerical simulations are carried out to verify the theoretical results in Section 4 and, finally, this work is summarized in Section 5.

\section{Local Stability and Existence of Local Hopf Bifurcation}

By direct computation, it can be concluded that if $R_{0}=((\mu+$ $\delta) p A \beta+(1-p) \beta \delta A+(\mu+\alpha) \delta \gamma) /(\mu+\alpha)(\mu+\gamma)(\mu+\delta)>1$, then system (3) has a unique positive equilibrium $E_{*}\left(S_{*}, I_{*}, R_{*}\right)$, where

$$
S_{*}=\frac{\mu+\alpha}{\beta},
$$

$$
\begin{gathered}
I_{*}=((\mu+\delta) p A \beta+(1-p) \beta \delta A+(\mu+\alpha) \delta \gamma \\
-(\mu+\alpha)(\mu+\gamma)(\mu+\delta)) \\
\times(\beta(\mu+\alpha)(\mu+\delta)-\alpha \beta \delta)^{-1}, \\
R_{*}=\frac{(1-p) A+\alpha I_{*}+\gamma S_{*} .}{\mu+\delta} .
\end{gathered}
$$

The characteristic equation of system (3) at $E_{*}$ is

$$
\begin{aligned}
& \left|\begin{array}{ccc}
\lambda-a_{11}-b_{11} e^{-\lambda \tau_{1}} & -b_{12} e^{-\lambda \tau_{1}} & -b_{13} e^{-\lambda \tau_{1}} \\
-b_{21} e^{-\lambda \tau_{1}} & \lambda-a_{22}-b_{22} e^{-\lambda \tau_{1}}-c_{22} e^{-\lambda \tau_{2}} & 0 \\
-a_{31} & -c_{32} e^{-\lambda \tau_{2}} & \lambda-a_{33}-b_{33} e^{-\lambda \tau_{1}}
\end{array}\right| \\
& =0,
\end{aligned}
$$

from which one can obtain

$$
\begin{aligned}
\lambda^{3}+ & A_{2} \lambda^{2}+A_{1} \lambda+A_{0}+\left(B_{2} \lambda^{2}+B_{1} \lambda+B_{0}\right) e^{-\lambda \tau_{1}} \\
& +\left(C_{2} \lambda^{2}+C_{1} \lambda+C_{0}\right) e^{-\lambda \tau_{2}}+\left(D_{1} \lambda+D_{0}\right) e^{-\lambda\left(\tau_{1}+\tau_{2}\right)} \\
& +\left(E_{1} \lambda+E_{0}\right) e^{-2 \lambda \tau_{1}}+F_{0} e^{-\lambda\left(2 \tau_{1}+\tau_{2}\right)}=0,
\end{aligned}
$$

where

$$
\begin{gathered}
A_{0}=-a_{11} a_{22} a_{33}, \quad A_{1}=a_{11} a_{22}+a_{11} a_{33}+a_{22} a_{33}, \\
A_{2}=-\left(a_{11}+a_{22}+a_{33}\right), \\
B_{0}=a_{22} a_{31} b_{13}-a_{11} a_{22} b_{33}-a_{11} a_{33} b_{22}-a_{22} a_{33} b_{11}, \\
B_{1}=b_{11}\left(a_{22}+a_{33}\right)+b_{22}\left(a_{11}+a_{33}\right) \\
+b_{33}\left(a_{11}+a_{22}\right)-a_{31} b_{13}, \\
B_{2}=-\left(b_{11}+b_{22}+b_{33}\right), \quad C_{0}=-a_{11} a_{33} c_{22}, \\
C_{1}=c_{22}\left(a_{11}+a_{33}\right), \\
C_{2}=-c_{22}, \quad D_{0}=c_{22}\left(a_{31} b_{13}-a_{11} b_{33}-a_{33} b_{11}\right), \\
D_{1}=c_{22}\left(b_{11}+b_{33}\right), \\
E_{1}=b_{33}\left(b_{11}+b_{22}\right), \quad F_{0}=-\left(b_{11} b_{33} c_{22}+b_{13} b_{21} c_{32}\right),
\end{gathered}
$$

with

$$
\begin{array}{crrr}
a_{11}=-(\mu+\gamma), & a_{22}=-\mu, & a_{31}=\gamma, & a_{33}-\mu, \\
b_{11}=-\beta I_{*}, & b_{12}=-\beta S_{*}, & b_{13}=\delta, & b_{21}=\beta I_{*}, \\
b_{22}=\beta S_{*}, & b_{33}=-\delta, & c_{22}=-\alpha, & c_{32}=\alpha .
\end{array}
$$


From the expressions of $b_{11}, b_{13}, b_{21}, b_{33}, c_{22}$, and $c_{32}$, one can obtain $F_{0}=0$. Therefore, (6) can be transformed into the following form:

$$
\begin{aligned}
\lambda^{3}+ & A_{2} \lambda^{2}+A_{1} \lambda+A_{0}+\left(B_{2} \lambda^{2}+B_{1} \lambda+B_{0}\right) e^{-\lambda \tau_{1}} \\
& +\left(C_{2} \lambda^{2}+C_{1} \lambda+C_{0}\right) e^{-\lambda \tau_{2}}+\left(D_{1} \lambda+D_{0}\right) e^{-\lambda\left(\tau_{1}+\tau_{2}\right)} \\
& +\left(E_{1} \lambda+E_{0}\right) e^{-2 \lambda \tau_{1}}=0 .
\end{aligned}
$$

Case $1\left(\tau_{1}=\tau_{2}=0\right)$. When $\tau_{1}=\tau_{2}=0$, (9) is equivalent to

$$
\lambda^{3}+A_{12} \lambda^{2}+A_{11} \lambda+A_{10}=0,
$$

where

$$
\begin{gathered}
A_{10}=A_{0}+B_{0}+C_{0}+D_{0}+E_{0}, \\
A_{11}=A_{1}+B_{1}+C_{1}+D_{1}+E_{1}, \\
A_{12}=A_{2}+B_{2}+C_{2} .
\end{gathered}
$$

It is easy to get that $A_{12}=2 \mu+\gamma+\delta+\beta I_{*}>0$. Therefore, according to the Routh-Hurwitz criterion, we can conclude that if $A_{12} A_{11}>A_{10}>0$, then the positive equilibrium $E_{*}$ of system (3) is locally asymptotically stable when $\tau_{1}=\tau_{2}=0$.

Case $2\left(\tau_{1}>0, \tau_{2}=0\right)$. When $\tau_{1}>0$ and $\tau_{2}=0$, (9) becomes the following:

$$
\begin{aligned}
\lambda^{3}+ & A_{22} \lambda^{2}+A_{21} \lambda+A_{20}+\left(B_{22} \lambda^{2}+B_{21} \lambda+B_{20}\right) e^{-\lambda \tau_{1}} \\
& +\left(E_{21} \lambda+E_{20}\right) e^{-2 \lambda \tau_{1}}=0,
\end{aligned}
$$

where

$$
\begin{array}{cc}
A_{20}=A_{0}+C_{0}, & A_{21}=A_{1}+C_{1}, \\
A_{22}=A_{2}+C_{2}, & B_{20}=B_{0}+D_{0}, \\
B_{21}=B_{1}+D_{1}, & B_{22}=B_{2}, \\
E_{20}=E_{0}, & E_{21}=E_{1} .
\end{array}
$$

Multiplying $e^{\lambda \tau_{1}}$ on both sides of (12), it is easy to get

$$
\begin{aligned}
B_{22} \lambda^{2} & +B_{21} \lambda+B_{20}+\left(\lambda^{3}+A_{22} \lambda^{2}+A_{21} \lambda+A_{20}\right) e^{\lambda \tau_{1}} \\
& +\left(E_{21} \lambda+E_{20}\right) e^{-\lambda \tau_{1}}=0 .
\end{aligned}
$$

Let $\lambda=i \omega_{1}\left(\omega_{1}>0\right)$ be the root of (14). Then,

$$
\begin{aligned}
& \left(A_{20}+E_{20}+A_{22} \omega_{1}^{2}\right) \cos \tau_{1} \omega_{1} \\
& \quad-\left(A_{21} \omega_{1}-E_{21} \omega_{1}-\omega_{1}^{3}\right) \sin \tau_{1} \omega_{1}=B_{22} \omega_{1}^{2}-B_{20}, \\
& \left(A_{20}-E_{20}+A_{22} \omega_{1}^{2}\right) \sin \tau_{1} \omega_{1} \\
& \quad+\left(A_{21} \omega_{1}+E_{21} \omega_{1}-\omega_{1}^{3}\right) \cos \tau_{1} \omega_{1}=-B_{21} \omega_{1} .
\end{aligned}
$$

Then, one can obtain

$$
\begin{aligned}
& \cos \tau_{1} \omega_{1}=\frac{p_{24} \omega_{1}^{4}+p_{22} \omega_{1}^{2}+p_{20}}{\omega_{1}^{6}+q_{24} \omega_{1}^{4}+q_{22} \omega_{1}^{2}+q_{20}}, \\
& \sin \tau_{1} \omega_{1}=\frac{p_{25} \omega_{1}^{5}+p_{23} \omega_{1}^{3}+p_{21} \omega_{1}}{\omega_{1}^{6}+q_{24} \omega_{1}^{4}+q_{22} \omega_{1}^{2}+q_{20}},
\end{aligned}
$$

where

$$
\begin{gathered}
p_{20}=B_{20}\left(E_{20}-A_{20}\right), \\
p_{21}=B_{20}\left(A_{21}+E_{21}\right)-B_{21}\left(A_{20}+E_{20}\right), \\
P_{22}=B_{22}\left(A_{20}-E_{20}\right)-B_{21}\left(A_{21}-E_{21}\right)+A_{22} B_{00}, \\
p_{23}=A_{22} B_{21}-B_{20}-B_{22}\left(A_{21}+E_{21}\right), \\
p_{24}=B_{21}-A_{22} B_{22}, \quad p_{25}=B_{22}, \quad q_{20}=A_{20}^{2}-E_{20}^{2}, \\
q_{22}=A_{21}^{2}-E_{21}^{2}-2 A_{20} A_{22}, \quad q_{24}=A_{22}^{2}-2 A_{21} .
\end{gathered}
$$

Since $\cos ^{2} \tau_{1} \omega_{1}+\sin ^{2} \tau_{1} \omega_{1}=1$, we have

$$
\omega_{1}^{12}+e_{25} \omega_{1}^{10}+e_{24} \omega_{1}^{8}+e_{23} \omega_{1}^{6}+e_{22} \omega_{1}^{4}+e_{21} \omega_{1}^{2}+e_{20}=0,
$$

where

$$
\begin{gathered}
e_{20}=q_{20}^{2}-p_{20}^{2}, \quad e_{21}=2 q_{20} q_{22}-2 p_{20} p_{22}-p_{21}^{2}, \\
e_{22}=q_{22}^{2}-p_{22}^{2}+2 q_{20} q_{24}-2 p_{20} p_{24}-2 p_{21} p_{23}, \\
e_{23}=2 q_{20}+2 q_{22} q_{24}-2 p_{22} p_{24}-2 p_{21} p_{25}-p_{23}^{2}, \\
e_{24}=q_{24}^{2}-p_{24}^{2}+2 q_{22}-2 p_{23} p_{25}, \quad e_{25}=2 q_{24}-p_{25}^{2} .
\end{gathered}
$$

Let $\omega_{1}^{2}=v_{1}$; then, (18) becomes

$$
v_{1}^{6}+e_{25} v_{1}^{5}+e_{24} v_{1}^{4}+e_{23} v_{1}^{3}+e_{22} v_{1}^{2}+e_{21} v_{1}+e_{20}=0 .
$$

In order to give the main results in the present paper, we make the following assumption.

$\left(H_{21}\right)$ Equation (20) has at least one positive real root.

If the condition $\left(\mathrm{H}_{21}\right)$ holds, then there exists a positive root $v_{10}$ of (20) which can make (14) have a pair of purely imaginary roots $\pm i \omega_{10}= \pm i \sqrt{v_{10}}$. For $\omega_{10}$, the corresponding critical value of delay is

$$
\tau_{10}=\frac{1}{\omega_{10}} \arccos \frac{p_{24} \omega_{10}^{4}+p_{22} \omega_{10}^{2}+p_{20}}{\omega_{10}^{6}+q_{24} \omega_{10}^{4}+q_{22} \omega_{10}^{2}+q_{20}} .
$$


Differentiating (14) with respect to $\tau_{1}$, we get

$$
\begin{aligned}
{\left[\frac{d \lambda}{d \tau_{1}}\right]^{-1}=} & -\left(2 B_{22} \lambda+B_{21}+\left(3 \lambda^{2}+2 A_{22} \lambda+A_{21}\right) e^{\lambda \tau_{1}}\right. \\
& \left.+E_{21} e^{-\lambda \tau_{1}}\right) \\
& \times\left(\left(E_{21} \lambda^{2}+E_{20} \lambda\right) e^{-\lambda \tau_{1}}\right. \\
& \left.-\left(\lambda^{4}+A_{22} \lambda^{3}+A_{21} \lambda^{2}+A_{20} \lambda\right) e^{\lambda \tau_{1}}\right)^{-1} \\
& -\frac{\tau_{1}}{\lambda} .
\end{aligned}
$$

Thus,

$$
\operatorname{Re}\left[\frac{d \lambda}{d \tau_{1}}\right]_{\tau_{1}=\tau_{10}}^{-1}=\frac{P_{2 R} Q_{2 R}+P_{2 I} Q_{2 I}}{Q_{2 R}^{2}+Q_{2 I}^{2}}
$$

where

$$
\begin{aligned}
P_{2 R}= & \left(A_{21}+E_{21}-3 \omega_{10}^{2}\right) \cos \tau_{10} \omega_{10}-2 A_{22} \omega_{10} \sin \tau_{10} \omega_{10} \\
& +B_{21}, \\
P_{2 I}= & \left(A_{21}-E_{21}-3 \omega_{10}^{2}\right) \sin \tau_{10} \omega_{10}+2 A_{22} \omega_{10} \cos \tau_{10} \omega_{10} \\
& +2 B_{22} \omega_{10}, \\
Q_{2 R}= & \left(A_{21} \omega_{10}^{2}-E_{21} \omega_{10}^{2}-\omega_{10}^{4}\right) \cos \tau_{10} \omega_{10} \\
& -\left(A_{22} \omega_{10}^{3}-A_{20} \omega_{10}-E_{20} \omega_{10}\right) \sin \tau_{10} \omega_{10}, \\
Q_{2 I}= & \left(A_{21} \omega_{10}^{2}+E_{21} \omega_{10}^{2}-\omega_{10}^{4}\right) \sin \tau_{10} \omega_{10} \\
& +\left(A_{22} \omega_{10}^{3}-A_{20} \omega_{10}+E_{20} \omega_{10}\right) \cos \tau_{10} \omega_{10} .
\end{aligned}
$$

It is obvious that if the condition $\left(H_{21}\right) P_{2 R} Q_{2 R}+P_{2 I} Q_{2 I} \neq$ 0 holds, then $\operatorname{Re}\left[d \lambda / d \tau_{1}\right]_{\tau=\tau_{10}}^{-1} \neq 0$. According to the Hopf bifurcation theorem in [17], the following results hold.

Theorem 1. If the conditions $\left(\mathrm{H}_{21}\right)-\left(\mathrm{H}_{22}\right)$ hold, the positive equilibrium $E_{*}\left(S_{*}, I_{*}, R_{*}\right)$ of system (3) is locally asymptotically stable for $\tau_{1} \in\left[0, \tau_{10}\right)$ and system (3) undergoes a Hopf bifurcation at the positive equilibrium $E_{*}\left(S_{*}, I_{*}, R_{*}\right)$ when $\tau_{1}=$ $\tau_{10}$.

Case $3\left(\tau_{1}=0, \tau_{2}>0\right)$. When $\tau_{1}=0$ and $\tau_{2}>0$, (9) becomes

$$
\lambda^{3}+A_{32} \lambda^{2}+A_{31} \lambda+A_{30}+\left(C_{32} \lambda^{2}+C_{31} \lambda+C_{30}\right) e^{-\lambda \tau_{2}}=0,
$$

where

$$
\begin{gathered}
A_{30}=A_{0}+B_{0}+E_{0}, \quad A_{31}=A_{1}+B_{1}+E_{1}, \\
A_{32}=A_{2}+B_{2}, \\
C_{30}=C_{0}+D_{0}, \quad C_{31}=C_{1}+D_{1}, \quad C_{32}=C_{2} .
\end{gathered}
$$

Let $\lambda=i \omega_{2}\left(\omega_{2}>0\right)$ be the root of (25). Then,

$$
\begin{gathered}
C_{31} \omega_{2} \sin \tau_{2} \omega_{2}+\left(C_{30}-C_{32} \omega_{2}^{2}\right) \cos \tau_{2} \omega_{2}=A_{32} \omega_{2}^{2}-A_{30}, \\
C_{31} \omega_{2} \cos \tau_{2} \omega_{2}-\left(C_{30}-C_{32} \omega_{2}^{2}\right) \sin \tau_{2} \omega_{2}=\omega_{2}^{3}-A_{31} \omega_{2},
\end{gathered}
$$

which follows that

$$
\omega_{2}^{6}+e_{32} \omega_{2}^{4}+e_{31} \omega_{2}^{2}+e_{30}=0
$$

with

$$
\begin{aligned}
& e_{30}=A_{30}^{2}-C_{30}^{2}, \\
& e_{31}=A_{31}^{2}-C_{31}^{2}-2 A_{30} A_{32}+2 C_{30} C_{32}, \\
& e_{32}=A_{32}^{2}-C_{32}^{2}-2 A_{31} .
\end{aligned}
$$

Let $\omega_{2}^{2}=v_{2}$; then, (28) becomes

$$
v_{2}^{3}+e_{32} v_{2}^{2}+e_{31} v_{2}+e_{30}=0
$$

Let

$$
f_{2}\left(v_{2}\right)=v_{2}^{3}+e_{32} v_{2}^{2}+e_{31} v_{2}+e_{30} .
$$

Discussion about the roots of (30) is similar to that in [18].

Lemma 2. (i) If $e_{30}<0$, then (30) has at least one positive root.

(ii) If $e_{30} \geq 0$ and $e_{32}^{2}-3 e_{31} \leq 0$, then (30) has no positive root.

(iii) If $e_{30} \geq 0$ and $e_{32}^{2}-3 e_{31}>0$, then (30) has positive root if and only if $v_{2}^{*}=\left(-e_{32}+\sqrt{e_{32}^{2}-3 e_{31}}\right) / 3>0$ and $f_{2}\left(v_{2}^{*}\right)<0$.

In what follows, we suppose that the coefficients in (30) satisfy the following condition:

$$
\begin{gathered}
\left(H_{31}\right):(\mathrm{a}) e_{30}<0 \text { or }(\mathrm{b}) e_{30} \geq 0, e_{32}^{2}-3 e_{31}>0, v_{2}^{*}=\left(-e_{32}+\right. \\
\left.\sqrt{e_{32}^{2}-3 e_{31}}\right) / 3>0, \text { and } f_{2}\left(v_{2}^{*}\right)<0 .
\end{gathered}
$$

If the condition $\left(\mathrm{H}_{31}\right)$ holds, we know that there exists a positive root $v_{20}$ of (30) such that (25) has a pair of purely imaginary roots $\pm i \omega_{20}= \pm i \sqrt{v_{20}}$. For $\omega_{20}$, the corresponding critical value of time delay is

$$
\begin{aligned}
& \tau_{20} \\
& =\frac{1}{\omega_{20}} \arccos \left(\left(\left(C_{31}-A_{32} C_{32}\right) \omega_{20}^{4}\right.\right. \\
& +\left(A_{30} C_{32}-A_{31} C_{31}+A_{32} C_{30}\right) \omega_{20}^{2} \\
& \left.-A_{30} C_{30}\right) \\
& \left.\times\left(C_{32} \omega_{20}^{4}+\left(C_{31}^{2}-2 C_{30} C_{32}\right) \omega_{20}^{2}+C_{30}^{2}\right)^{-1}\right) .
\end{aligned}
$$

Differentiating two sides of (25) with respect to $\tau_{2}$, we have

$$
\begin{aligned}
{\left[\frac{d \lambda}{d \tau_{2}}\right]^{-1}=} & -\frac{3 \lambda^{2}+2 A_{32} \lambda+A_{31}}{\lambda\left(\lambda^{3}+A_{32} \lambda^{2}+A_{31} \lambda+A_{30}\right)} \\
& +\frac{2 C_{32} \lambda+C_{31}}{\lambda\left(C_{32} \lambda^{2}+C_{31} \lambda+C_{30}\right)}-\frac{\tau_{2}}{\lambda} .
\end{aligned}
$$


Thus,

$$
\begin{aligned}
\operatorname{Re}\left[\frac{d \lambda}{d \tau_{2}}\right]_{\tau_{2}=\tau_{20}}^{-1} & \\
& =\frac{f_{2}^{\prime}\left(v_{2 *}\right)}{\left(B_{21} \omega_{10}-B_{23} \omega_{10}^{3}\right)^{2}+\left(B_{24} \omega_{20}^{4}-B_{22} \omega_{20}^{2}+B_{20}\right)^{2}}
\end{aligned}
$$

where $f_{2}\left(v_{2}\right)=v_{2}^{3}+e_{32} v_{2}^{2}+e_{31} v_{2}+e_{30}$ and $v_{2 *}=\omega_{20}^{2}$.

Obviously, if the condition $\left(H_{32}\right) f_{2}^{\prime}\left(v_{2 *}\right) \neq 0$ holds, then $\operatorname{Re}\left[d \lambda / d \tau_{2}\right]_{\tau_{2}=\tau_{20}}^{-1} \neq 0$. According to the Hopf bifurcation theorem in [17], the following results hold.

Theorem 3. If the conditions $\left(H_{31}\right)-\left(H_{32}\right)$ hold, the positive equilibrium $E_{*}\left(S_{*}, I_{*}, R_{*}\right)$ of system (3) is locally asymptotically stable for $\tau_{2} \in\left[0, \tau_{20}\right)$ and system (3) undergoes a Hopf bifurcation at $E_{*}\left(S_{*}, I_{*}, R_{*}\right)$ when $\tau_{2}=\tau_{20}$.

Case $4\left(\tau_{1}>0, \tau_{2}>0, \tau_{2} \in\left(0, \tau_{20}\right)\right)$. We consider (9) with $\tau_{2}$ in its stable interval and choose $\tau_{1}$ as a bifurcation parameter. Multiplying by $e^{\lambda \tau_{1}},(9)$ becomes

$$
\begin{aligned}
B_{2} \lambda^{2} & +B_{1} \lambda+B_{0}+\left(\lambda^{3}+A_{2} \lambda^{2}+A_{1} \lambda+A_{0}\right) e^{\lambda \tau_{1}} \\
& +\left(E_{1} \lambda+E_{0}\right) e^{-\lambda \tau_{1}}+\left(D_{1} \lambda+D_{0}\right) e^{-\lambda \tau_{2}} \\
& +\left(C_{2} \lambda^{2}+C_{1} \lambda+C_{0}\right) e^{\lambda\left(\tau_{1}-\tau_{2}\right)}=0
\end{aligned}
$$

Let $\lambda=i \omega_{1 *}\left(\omega_{1 *}>0\right)$ be the root of (35). Then,

$$
\begin{aligned}
& M_{41} \cos \tau_{1} \omega_{1 *}-M_{42} \sin \tau_{1} \omega_{1 *}=M_{43} \\
& M_{44} \sin \tau_{1} \omega_{1 *}+M_{45} \cos \tau_{1} \omega_{1 *}=M_{46}
\end{aligned}
$$

where

$$
\begin{aligned}
M_{41}= & A_{0}+E_{0}-A_{2} \omega_{1 *}^{2}+\left(C_{0}-C_{2} \omega_{1 *}^{2}\right) \cos \tau_{2} \omega_{1 *} \\
& +C_{1} \omega_{1 *} \sin \tau_{2} \omega_{1 *}, \\
M_{42}= & A_{1} \omega_{1 *}-E_{1} \omega_{1 *}-\omega_{1 *}^{3}-\left(C_{0}-C_{2} \omega_{1 *}^{2}\right) \sin \tau_{2} \omega_{1 *} \\
& +C_{1} \omega_{1 *} \cos \tau_{2} \omega_{1 *}, \\
M_{43}= & B_{2} \omega_{1 *}^{2}-B_{0}-D_{1} \omega_{1 *} \sin \tau_{2} \omega_{1 *}-D_{0} \cos \tau_{2} \omega_{1 *}, \\
M_{44}= & A_{0}-E_{0}-A_{2} \omega_{1 *}^{2}+\left(C_{0}-C_{2} \omega_{1 *}^{2}\right) \cos \tau_{2} \omega_{1 *} \\
& +C_{1} \omega_{1 *} \sin \tau_{2} \omega_{1 *}, \\
M_{45}= & A_{1} \omega_{1 *}+E_{1} \omega_{1 *}-\omega_{1 *}^{3}-\left(C_{0}-C_{2} \omega_{1 *}^{2}\right) \sin \tau_{2} \omega_{1 *} \\
& +C_{1} \omega_{1 *} \cos \tau_{2} \omega_{1 *}, \\
M_{46}= & -B_{1} \omega_{1 *}+D_{0} \sin \tau_{2} \omega_{1 *}-D_{1} \omega_{1 *} \cos \tau_{2} \omega_{1 *} \cdot
\end{aligned}
$$

Then, we can obtain

$$
\begin{aligned}
& \cos \tau_{1} \omega_{1 *} \\
& =\frac{h_{40}\left(\omega_{1 *}\right)+h_{41}\left(\omega_{1 *}\right) \cos \tau_{2} \omega_{1 *}+h_{42}\left(\omega_{1 *}\right) \sin \tau_{2} \omega_{1 *}}{g_{40}\left(\omega_{1 *}\right)+g_{41}\left(\omega_{1 *}\right) \cos \tau_{2} \omega_{1 *}+g_{42}\left(\omega_{1 *}\right) \sin \tau_{2} \omega_{1 *}} \\
& \sin \tau_{1} \omega_{1 *} \\
& =\frac{h_{40}^{\prime}\left(\omega_{1 *}\right)+h_{41}^{\prime}\left(\omega_{1 *}\right) \cos \tau_{2} \omega_{1 *}+h_{42}^{\prime}\left(\omega_{1 *}\right) \sin \tau_{2} \omega_{1 *}}{g_{40}\left(\omega_{1 *}\right)+g_{41}\left(\omega_{1 *}\right) \cos \tau_{2} \omega_{1 *}+g_{42}\left(\omega_{1 *}\right) \sin \tau_{2} \omega_{1 *}},
\end{aligned}
$$

where

$$
\begin{aligned}
& g_{40}\left(\omega_{1 *}\right)=\omega_{1 *}^{6}+\left(A_{2}^{2}+C_{2}^{2}-2 A_{1}\right) \omega_{1 *}^{4} \\
& +\left(A_{1}^{2}+C_{1}^{2}-E_{1}^{2}-2 A_{0} A_{2}-2 C_{0} C_{2}\right) \omega_{1 *}^{2} \\
& +A_{0}^{2}+C_{0}^{2}-C_{0}^{2} \\
& g_{41}\left(\omega_{1 *}\right)=2\left(A_{2} C_{2}-C_{1}\right) \omega_{1 *}^{4} \\
& +2\left(A_{1} C_{1}-A_{0} C_{2}-A_{2} C_{0}\right) \omega_{1 *}^{2}+2 A_{0} C_{0}, \\
& g_{42}\left(\omega_{1 *}\right)=-2 C_{2} \omega_{1 *}^{5}+2\left(A_{1} C_{2}-A_{2} C_{1}-C_{0}\right) \omega_{1 *}^{3} \\
& +2\left(A_{0} C_{1}-A_{1} C_{0}\right) \omega_{1 *}, \\
& h_{40}\left(\omega_{1 *}\right)=\left(B_{1}+A_{2} B_{2}\right) \omega_{1 *}^{4} \\
& +\left[A_{2} B_{0}-C_{1} D_{1}+C_{2} D_{0}-B_{1}\left(A_{1}-E_{1}\right)\right. \\
& \left.+B_{2}\left(A_{0}-E_{0}\right)\right] \omega_{1 *}^{2} \\
& +B_{0}\left(E_{0}-A_{0}\right)-C_{0} D_{0} \text {, } \\
& h_{41}\left(\omega_{1 *}\right)=\left(D_{1}-B_{2} C_{2}\right) \omega_{1 *}^{4} \\
& +\left[A_{2} D_{0}+B_{0} C_{2}-B_{1} C_{1}\right. \\
& \left.+B_{2} C_{0}+D_{1}\left(E_{1}-A_{1}\right)\right] \omega_{1 *}^{2} \\
& +D_{0}\left(E_{0}-A_{0}\right)-B_{0} C_{0} \text {, } \\
& h_{42}\left(\omega_{1 *}\right)=\left(A_{2} D_{1}-B_{1} C_{2}+B_{2} C_{1}-D_{0}\right) \omega_{1 *}^{3} \\
& +\left[D_{0}\left(A_{1}-E_{1}\right)-D_{1}\left(A_{0}-E_{0}\right)\right. \\
& \left.+B_{1} C_{0}-B_{0} C_{1}\right] \omega_{1 *}, \\
& h_{40}^{\prime}\left(\omega_{1 *}\right)=B_{2} \omega_{1 *}^{5} \\
& +\left[A_{2} B_{1}+C_{2} D_{1}-B_{0}-B_{2}\left(A_{1}+E_{1}\right)\right] \omega_{1 *}^{3} \\
& +\left[B_{0}\left(A_{1}+E_{1}\right)-B_{1}\left(A_{0}+E_{0}\right)\right. \\
& \left.-C_{0} D_{1}+C_{1} D_{0}\right] \omega_{1 *}, \\
& h_{41}^{\prime}\left(\omega_{1 *}\right)=\left(A_{2} D_{1}+B_{1} C_{2}-B_{2} C_{1}-D_{0}\right) \omega_{1 *}^{3} \\
& +\left[D_{0}\left(A_{1}+E_{1}\right)-D_{1}\left(A_{0}+E_{0}\right)\right. \\
& \left.+B_{0} C_{1}-B_{1} C_{0}\right] \omega_{1 *},
\end{aligned}
$$




$$
\begin{aligned}
h_{42}^{\prime}\left(\omega_{1 *}\right)= & -\left(B_{2} C_{2}+D_{1}\right) \omega_{1 *}^{4} \\
& +\left[B_{0} C_{2}-B_{1} C_{1}-A_{2} D_{0}+B_{2} C_{0}\right. \\
& \left.\quad+D_{1}\left(A_{1}+E_{1}\right)\right] \omega_{1 *}^{2} \\
& +D_{0}\left(A_{0}+E_{0}\right)-B_{0} C_{0} .
\end{aligned}
$$

Then, we can get a function with respect to $\omega_{1 *}$ :

$$
\cos ^{2} \tau_{1} \omega_{1 *}+\sin ^{2} \tau_{1} \omega_{1 *}=1
$$

Next, we suppose that $\left(H_{41}\right):(40)$ has at least one positive real root.

If the condition $\left(H_{41}\right)$ holds, then there exists a $\omega_{10}^{*}$ such that (35) has a pair of purely imaginary roots $\pm i \omega_{10}^{*}$. For $\omega_{10}^{*}$, the corresponding critical value of time delay is

$$
\begin{aligned}
\tau_{10}^{*}=\frac{1}{\omega_{10}^{*}} \arccos ( & \left(h_{40}\left(\omega_{10}^{*}\right)+h_{41}\left(\omega_{10}^{*}\right) \cos \tau_{2} \omega_{10}^{*}\right. \\
+ & \left.h_{42}\left(\omega_{10}^{*}\right) \sin \tau_{2} \omega_{10}^{*}\right) \\
\times & \left(g_{40}\left(\omega_{10}^{*}\right)+g_{41}\left(\omega_{10}^{*}\right) \cos \tau_{2} \omega_{10}^{*}\right. \\
& \left.\left.+g_{42}\left(\omega_{10}^{*}\right) \sin \tau_{2} \omega_{10}^{*}\right)^{-1}\right) .
\end{aligned}
$$

Taking the derivative with respect to $\tau_{1}$ in (35), we get

$$
\left[\frac{d \lambda}{d \tau_{1}}\right]^{-1}=\frac{g_{41}(\lambda)}{g_{42}(\lambda)}-\frac{\tau_{1}}{\lambda},
$$

where

$$
\begin{aligned}
g_{41}(\lambda)= & 2 B_{2} \lambda+B_{1}+E_{1} e^{-\lambda \tau_{1}}+\left(3 \lambda^{2}+2 A_{2} \lambda+A_{1}\right) e^{\lambda \tau_{1}} \\
& -\left(\tau_{2} D_{1} \lambda-D_{1}+\tau_{2} D_{0}\right) e^{-\lambda \tau_{2}} \\
& -\left[\tau_{2} C_{2} \lambda^{2}-\left(2 C_{2}-\tau_{2} C_{1}\right) \lambda+C_{1}-\tau_{2} C_{0}\right] e^{\lambda\left(\tau_{1}-\tau_{2}\right)} \\
g_{42}(\lambda)= & \left(E_{1} \lambda^{2}+E_{0} \lambda\right) e^{-\lambda \tau_{1}} \\
& -\left(\lambda^{4}+A_{2} \lambda^{3}+A_{1} \lambda^{2}+A_{0} \lambda\right) e^{\lambda \tau_{1}} \\
& -\left(C_{2} \lambda^{3}+C_{1} \lambda^{2}+C_{0} \lambda\right) e^{\lambda\left(\tau_{1}-\tau_{2}\right)}
\end{aligned}
$$

Thus,

$$
\operatorname{Re}\left[\frac{d \lambda}{d \tau_{1}}\right]_{\tau_{1}=\tau_{10}^{*}}^{-1}=\frac{P_{4 R} Q_{4 R}+P_{4 I} Q_{4 I}}{Q_{4 R}^{2}+Q_{4 I}^{2}}
$$

where

$$
\begin{aligned}
& P_{4 R}=B_{1}+\left(D_{1}-\tau_{2} D_{0}\right) \cos \tau_{2} \omega_{10}^{*}-\tau_{2} D_{1} \omega_{10}^{*} \sin \tau_{2} \omega_{10}^{*} \\
& +\left[A_{1}+E_{1}-3\left(\omega_{10}^{*}\right)^{2}\right. \\
& +\left(\tau_{2} C_{2}\left(\omega_{10}^{*}\right)^{2}+\tau_{2} C_{0}-C_{1}\right) \cos \tau_{2} \omega_{10}^{*} \\
& \left.+\left(2 C_{2}-\tau_{2} C_{1}\right) \sin \tau_{2} \omega_{10}^{*}\right] \cos \tau_{10}^{*} \omega_{10}^{*} \\
& +\left[\left(\tau_{2} C_{2}\left(\omega_{10}^{*}\right)^{2}+\tau_{2} C_{0}-C_{1}\right) \sin \tau_{2} \omega_{10}^{*}\right. \\
& \left.-\left(2 C_{2}-\tau_{2} C_{1}\right)-2 A_{2} \omega_{10}^{*}\right] \sin \tau_{10}^{*} \omega_{10}^{*}, \\
& P_{4 I}=2 B_{2} \omega_{10}^{*}-\left(D_{1}-\tau_{2} D_{0}\right) \sin \tau_{2} \omega_{10}^{*}-\tau_{1} D_{1} \omega_{10}^{*} \cos \tau_{2} \omega_{10}^{*} \\
& +\left[A_{1}-E_{1}-3\left(\omega_{10}^{*}\right)^{2}\right. \\
& +\left(\tau_{2} C_{2}\left(\omega_{10}^{*}\right)^{2}+\tau_{2} C_{0}-C_{1}\right) \cos \tau_{2} \omega_{10}^{*} \\
& \left.+\left(2 C_{2}-\tau_{2} C_{1}\right) \sin \tau_{2} \omega_{10}^{*}\right] \sin \tau_{10}^{*} \omega_{10}^{*} \\
& +\left[2 A_{2} \omega_{10}^{*}-\left(\tau_{2} C_{2}\left(\omega_{10}^{*}\right)^{2}+\tau_{2} C_{0}-C_{1}\right) \cos \tau_{2} \omega_{10}^{*}\right. \\
& \left.+\left(2 C_{2}-\tau_{2} C_{1}\right) \sin \tau_{2} \omega_{10}^{*}\right] \cos \tau_{10}^{*} \omega_{10}^{*}, \\
& Q_{4 R}=\left[A_{0} \omega_{10}^{*}+E_{0} \omega_{10}^{*}-A_{2}\left(\omega_{10}^{*}\right)^{3}+C_{1}\left(\omega_{10}^{*}\right)^{2} \sin \tau_{2} \omega_{10}^{*}\right. \\
& \left.-\left(C_{2}\left(\omega_{10}^{*}\right)^{3}-C_{0} \omega_{10}^{*}\right) \cos \tau_{2} \omega_{10}^{*}\right] \sin \tau_{10}^{*} \omega_{10}^{*} \\
& +\left[A_{1}\left(\omega_{10}^{*}\right)^{2}-E_{1}\left(\omega_{10}^{*}\right)^{2}-\left(\omega_{10}^{*}\right)^{4}\right. \\
& +C_{1}\left(\omega_{10}^{*}\right)^{2} \cos \tau_{2} \omega_{10}^{*} \\
& \left.+\left(C_{2}\left(\omega_{10}^{*}\right)^{3}-C_{0} \omega_{10}^{*}\right) \sin \tau_{2} \omega_{10}^{*}\right] \cos \tau_{10}^{*} \omega_{10}^{*} \text {, } \\
& Q_{4 I}=\left[A_{2}\left(\omega_{10}^{*}\right)^{3}-A_{0} \omega_{10}^{*}+E_{0} \omega_{10}^{*}-C_{1}\left(\omega_{10}^{*}\right)^{2} \sin \tau_{2} \omega_{10}^{*}\right. \\
& \left.+\left(C_{2}\left(\omega_{10}^{*}\right)^{3}-C_{0} \omega_{10}^{*}\right) \cos \tau_{2} \omega_{10}^{*}\right] \cos \tau_{10}^{*} \omega_{10}^{*} \\
& +\left[A_{1}\left(\omega_{10}^{*}\right)^{2}+E_{1}\left(\omega_{10}^{*}\right)^{2}-\left(\omega_{10}^{*}\right)^{4}\right. \\
& +C_{1}\left(\omega_{10}^{*}\right)^{2} \cos \tau_{2} \omega_{10}^{*} \\
& \left.+\left(C_{2}\left(\omega_{10}^{*}\right)^{3}-C_{0} \omega_{10}^{*}\right) \sin \tau_{2} \omega_{10}^{*}\right] \sin \tau_{10}^{*} \omega_{10}^{*} \text {. }
\end{aligned}
$$

Thus, if the condition $\left(H_{42}\right) P_{4 R} Q_{4 R}+P_{4 I} Q_{4 I} \neq 0$ holds, then $\operatorname{Re}[d \lambda / d \tau]_{\tau_{1}=\tau_{10}^{*}}^{-1} \neq 0$, which implies that the transversality condition is satisfied. According to the Hopf bifurcation theorem in [17], we can conclude the discussions above as follows.

Theorem 4. If the conditions $\left(H_{41}\right)-\left(H_{42}\right)$ hold and $\tau_{2} \in$ $\left(0, \tau_{20}\right)$, the positive equilibrium $E_{*}\left(S_{*}, I_{*}, R_{*}\right)$ of system (3) is locally asymptotically stable for $\tau_{1} \in\left[0, \tau_{10}^{*}\right)$ and system (3) undergoes a Hopf bifurcation at $E_{*}\left(S_{*}, I_{*}, R_{*}\right)$ when $\tau_{1}=\tau_{10}^{*}$. 

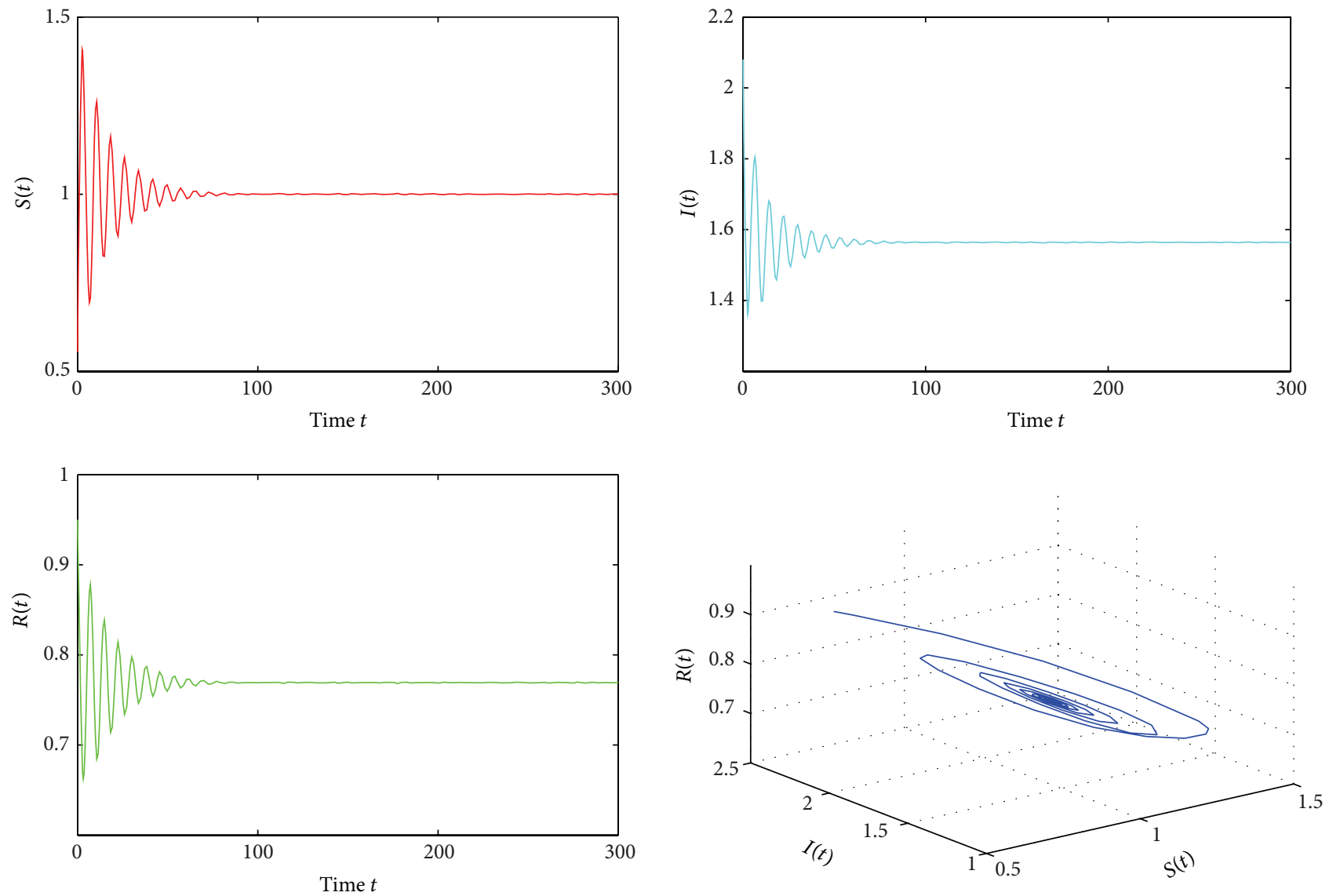

FIGURE 1: $E_{*}$ is locally asymptotically stable for $\tau_{1}=2.3750<\tau_{10}=2.8957$.

Case $5\left(\tau_{1}>0, \tau_{2}>0\right.$ and $\left.\tau_{1} \in\left(0, \tau_{10}\right)\right)$. We consider (9) with $\tau_{1}$ in its stable interval and $\tau_{2}$ is considered as a bifurcation parameter.

Let $\lambda=i \omega_{2 *}\left(\omega_{2 *}>0\right)$ be the root of (9). Then,

$$
\begin{aligned}
& M_{51} \sin \tau_{2} \omega_{2 *}+M_{52} \cos \tau_{2} \omega_{2 *}=M_{53} \\
& M_{51} \cos \tau_{2} \omega_{2 *}-M_{52} \sin \tau_{2} \omega_{2 *}=M_{54}
\end{aligned}
$$

where

$$
\begin{aligned}
M_{51}= & C_{1} \omega_{2 *}-D_{0} \sin \tau_{1} \omega_{2 *}+D_{1} \omega_{2 *} \cos \tau_{1} \omega_{2 *}, \\
M_{52}= & C_{0}-C_{2} \omega_{2 *}^{2}+D_{0} \cos \tau_{1} \omega_{2 *}+D_{1} \omega_{2 *} \sin \tau_{1} \omega_{2 *}, \\
M_{53}= & A_{2} \omega_{2 *}^{2}-A_{0}-B_{1} \omega_{2 *} \sin \tau_{1} \omega_{2 *} \\
& -\left(B_{0}-B_{2} \omega_{2 *}^{2}\right) \cos \tau_{1} \omega_{2 *} \\
& -E_{1} \omega_{2 *} \sin 2 \tau_{1} \omega_{2 *}-E_{0} \cos 2 \tau_{1} \omega_{2 *}, \\
M_{54}= & \omega_{2 *}^{3}-A_{1} \omega_{2 *}-B_{1} \omega_{2 *} \cos \tau_{1} \omega_{2 *} \\
& +\left(B_{0}-B_{2} \omega_{2 *}^{2}\right) \sin \tau_{1} \omega_{2 *} \\
& -E_{1} \omega_{2 *} \cos 2 \tau_{1} \omega_{2 *}+E_{0} \sin 2 \tau_{1} \omega_{2 *} .
\end{aligned}
$$

Then, we have

$$
\begin{aligned}
& g_{50}\left(\omega_{2 *}\right)+g_{51}\left(\omega_{2 *}\right) \cos \tau_{1} \omega_{2 *}+g_{52}\left(\omega_{2 *}\right) \sin \tau_{1} \omega_{2 *} \\
& +g_{53}\left(\omega_{2 *}\right) \cos 2 \tau_{1} \omega_{2 *}+g_{54}\left(\omega_{2 *}\right) \sin 2 \tau_{1} \omega_{2 *}=0,
\end{aligned}
$$

where

$$
\begin{aligned}
& g_{50}\left(\omega_{2 *}\right)=\omega_{2 *}^{6}+\left(A_{2}^{2}+B_{2}^{2}-C_{2}^{2}-2 A_{1}\right) \omega_{2 *}^{4} \\
& +\left(A_{1}^{2}+B_{1}^{2}-C_{1}^{2}-D_{1}^{2}-E_{1}^{2}-2 A_{0} A_{2}\right. \\
& \left.-2 B_{0} B_{2}+2 C_{0} C_{2}\right) \omega_{2 *}^{2} \\
& +A_{0}^{2}-C_{0}^{2}-D_{0}^{2}+E_{0}^{2} \\
& g_{51}\left(\omega_{2 *}\right)=2\left(A_{2} B_{2}-B_{1}\right) \omega_{2 *}^{4} \\
& +2\left(A_{1} B_{1}-A_{0} B_{2}-A_{2} B_{0}-C_{1} D_{1}+C_{2} D_{0}\right) \omega_{2 *}^{2} \\
& +2\left(A_{0} B_{0}-C_{0} D_{0}\right) \\
& g_{52}\left(\omega_{2 *}\right)=-2 B_{2} \omega_{2 *}^{5}+2\left(A_{1} B_{2}-A_{2} B_{1}+C_{2} D_{1}+B_{0}\right) \omega_{2 *}^{3} \\
& +2\left(A_{0} B_{1}-A_{1} B_{0}-C_{0} D_{1}+C_{1} D_{0}\right) \omega_{2 *},
\end{aligned}
$$



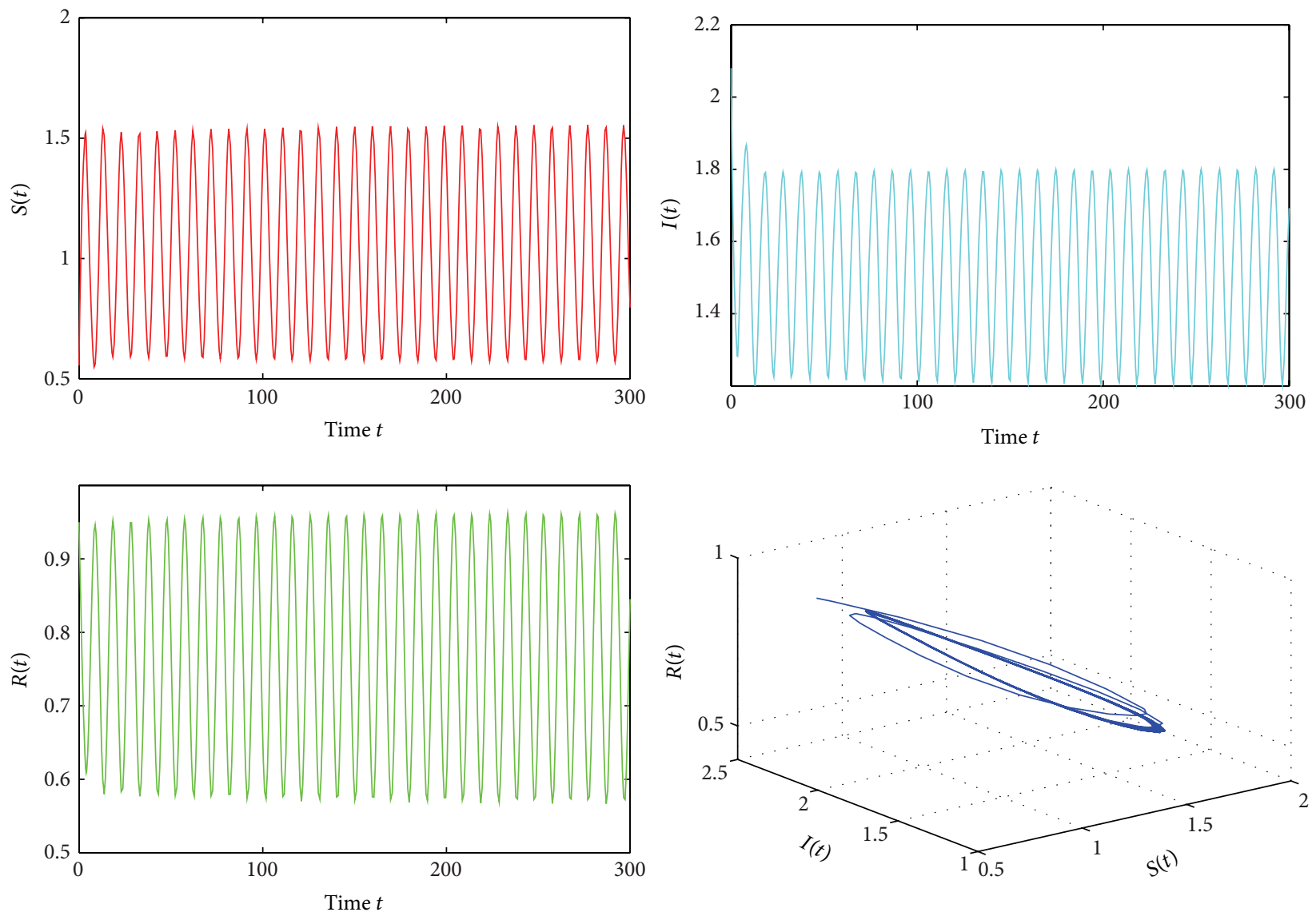

FIgURe 2: $E_{*}$ is unstable for $\tau_{1}=3.2950>\tau_{10}=2.8957$.

$g_{53}\left(\omega_{2 *}\right)=-2 E_{1} \omega_{2 *}^{4}+2\left(A_{1} E_{1}-A_{2} E_{0}\right) \omega_{2 *}^{2}+2 A_{0} E_{0}$,

$g_{54}\left(\omega_{2 *}\right)=2\left(E_{0}-A_{2} E_{1}\right) \omega_{2 *}^{3}+2\left(A_{0} E_{1}-A_{1} E_{0}\right) \omega_{2 *}$.

Similar to Case 4 , we suppose that $\left(H_{51}\right)$ : (48) has at least one positive real root. If the condition $\left(H_{51}\right)$ holds, then there exists a $\omega_{20}^{*}$ such that (9) has a pair of purely imaginary roots $\pm i \omega_{20}^{*}$. For $\omega_{20}^{*}$, the corresponding critical value of time delay is

$$
\tau_{20}^{*}=\left.\frac{1}{\omega_{20}^{*}} \arccos \frac{M_{51} \times M_{54}+M_{52} \times M_{53}}{M_{51}^{2}+M_{52}^{2}}\right|_{\tau_{2}=\tau_{20}^{*}} .
$$

Differentiating (9) with respect to $\tau_{2}$, we have

$$
\left[\frac{d \lambda}{d \tau_{2}}\right]^{-1}=\frac{g_{51}(\lambda)}{g_{52}(\lambda)}-\frac{\tau_{2}}{\lambda}
$$

where

$$
\begin{aligned}
g_{51}(\lambda)= & 3 \lambda^{2}+2 A_{2} \lambda+A_{1} \\
& +\left[\left(2 B_{2}-\tau_{1} B_{1}\right) \lambda-\tau_{1} B_{2} \lambda^{2}+B_{1}-\tau_{1} B_{0}\right] e^{-\lambda \tau_{1}} \\
& +\left(2 C_{2} \lambda+C_{1}\right) e^{-\lambda \tau_{2}} \\
& +\left(D_{1}-\tau_{1} D_{0}-\tau_{1} D_{1} \lambda\right) e^{-\lambda\left(\tau_{1}+\tau_{2}\right)} \\
& +\left(E_{1}-2 \tau_{1} E_{0}-2 \tau_{1} E_{1} \lambda\right) e^{-2 \lambda \tau_{1}} \\
g_{52}(\lambda)= & \left(C_{2} \lambda^{3}+C_{1} \lambda^{2}+C_{0} \lambda\right) e^{-\lambda \tau_{2}} \\
& +\left(D_{1} \lambda^{2}+D_{0} \lambda\right) e^{-\lambda\left(\tau_{1}+\tau_{2}\right)}
\end{aligned}
$$

Define

$$
\operatorname{Re}\left[\frac{d \lambda}{d \tau_{2}}\right]_{\tau_{2}=\tau_{20}^{*}}^{-1}=\frac{P_{5 R} Q_{5 R}+P_{5 I} Q_{5 I}}{Q_{5 R}^{2}+Q_{5 I}^{2}} .
$$

If the condition $\left(H_{52}\right) P_{5 R} Q_{5 R}+P_{5 I} Q_{5 I} \neq 0$ holds, then $\operatorname{Re}\left[d \lambda / d \tau_{1}\right]_{\tau_{2}=\tau_{20}^{*}}^{-1} \neq 0$. Therefore, according to the Hopf bifurcation theorem in [17], we can conclude the discussions above as follows. 

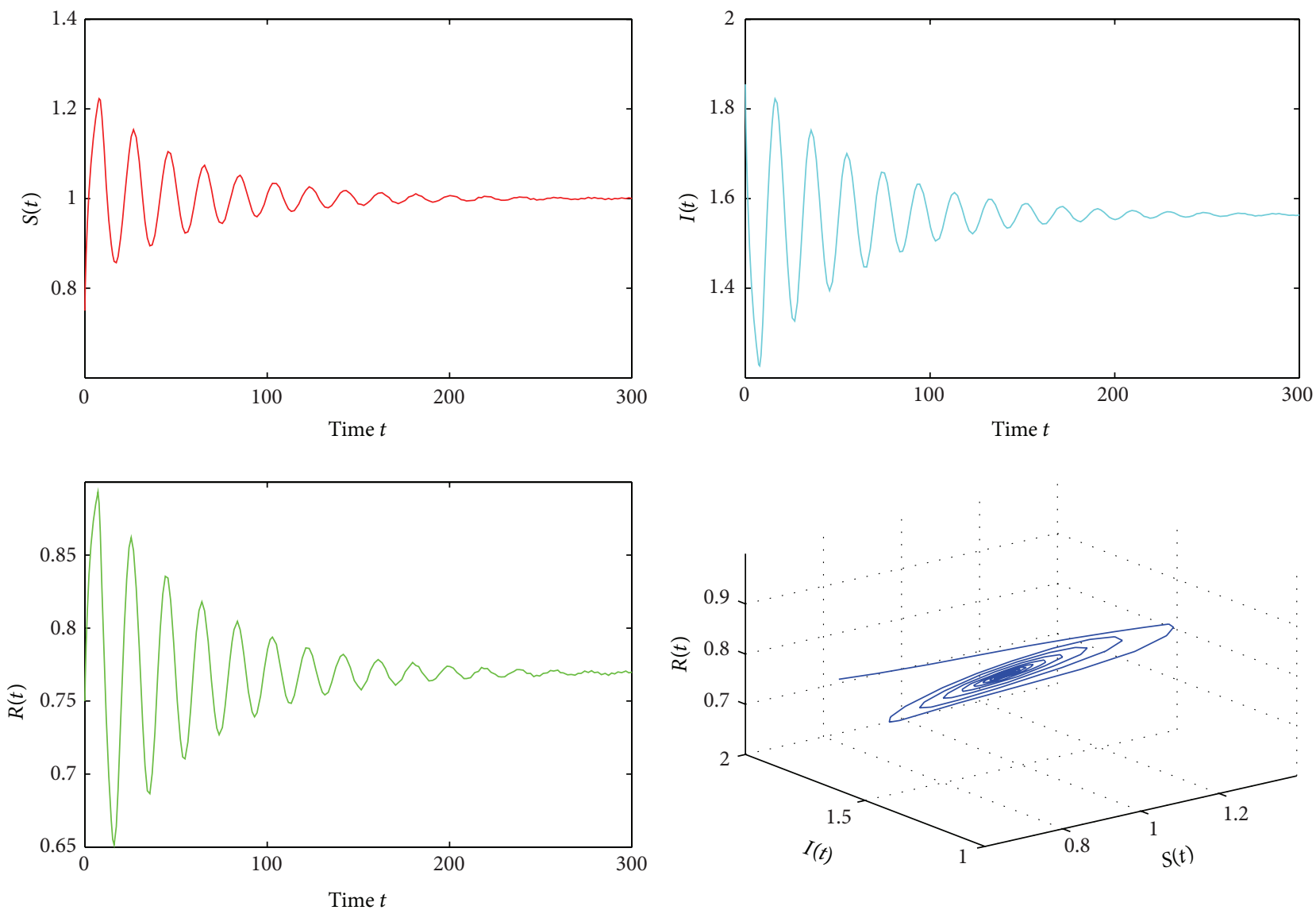

FIGURE 3: $E_{*}$ is locally asymptotically stable for $\tau_{2}=7.2500<\tau_{20}=8.4669$.

Theorem 5. If the conditions $\left(H_{51}\right)-\left(H_{52}\right)$ hold and $\tau_{1} \in$ $\left(0, \tau_{10}\right)$, the positive equilibrium $E_{*}\left(S_{*}, I_{*}, R_{*}\right)$ of system (3) is locally asymptotically stable for $\tau_{2} \in\left[0, \tau_{20}^{*}\right)$ and system (3) undergoes a Hopf bifurcation at $E_{*}\left(S_{*}, I_{*}, R_{*}\right)$ when $\tau_{2}=$ $\tau_{20}^{*}$.

\section{Direction and Stability of the Hopf Bifurcation}

In this section, we determine the properties of the Hopf bifurcation of system (3) with respect to $\tau_{2}$ for $\tau_{1} \in\left(0, \tau_{10}\right)$. Throughout this section, we assume that $\tau_{1 *}<\tau_{20}^{*}$, where $\tau_{1} \epsilon$ $\left(0, \tau_{10}\right)$.

Let $\tau_{2}=\tau_{20}^{*}+\mu, \mu \in R$; then, $\mu=0$ is the Hopf bifurcation value of system (3). Rescale the time delay $t \rightarrow\left(t / \tau_{2}\right)$. Let $u_{1}(t)=S(t)-S_{*}$, let $u_{2}(t)=I(t)-I_{*}$, and let $u_{3}(t)=R(t)-$ $R_{*}$; then, system (3) can be transformed into an FDE in $C=$ $C\left([-1,0], R^{3}\right)$ :

$$
\dot{u}(t)=L_{\mu} u_{t}+F\left(\mu, u_{t}\right)
$$

where $u(t)=\left(u_{1}(t), u_{2}(t), u_{3}(t)\right)^{T}$ and $L_{\mu}: C \rightarrow R^{3}$ and $F:$ $R \times C \rightarrow R^{3}$ are given, respectively, by

$$
\begin{gathered}
L_{\mu} \phi=\left(\tau_{20}^{*}+\mu\right)\left(A^{\prime} \phi(0)+B^{\prime} \phi\left(-\frac{\tau_{1 *}}{\tau_{20}^{*}}\right)+C^{\prime} \phi(-1)\right), \\
F(\mu, \phi)=\left(\tau_{20}^{*}+\mu\right)\left(\begin{array}{c}
-\beta \phi_{1}\left(-\frac{\tau_{1 *}}{\tau_{20}^{*}}\right) \phi_{2}\left(-\frac{\tau_{1 *}}{\tau_{20}^{*}}\right) \\
\beta \phi_{1}\left(-\frac{\tau_{1 *}}{\tau_{20}^{*}}\right) \phi_{2}\left(-\frac{\tau_{1 *}}{\tau_{20}^{*}}\right) \\
0
\end{array}\right),
\end{gathered}
$$

with

$$
\begin{gathered}
A^{\prime}=\left(\begin{array}{ccc}
a_{11} & 0 & 0 \\
0 & a_{22} & 0 \\
a_{31} & 0 & a_{33}
\end{array}\right), \quad B^{\prime}=\left(\begin{array}{ccc}
b_{11} & b_{12} & b_{13} \\
b_{21} & b_{22} & 0 \\
0 & 0 & b_{33}
\end{array}\right), \\
C^{\prime}=\left(\begin{array}{ccc}
0 & 0 & 0 \\
0 & c_{22} & 0 \\
0 & c_{32} & 0
\end{array}\right) .
\end{gathered}
$$



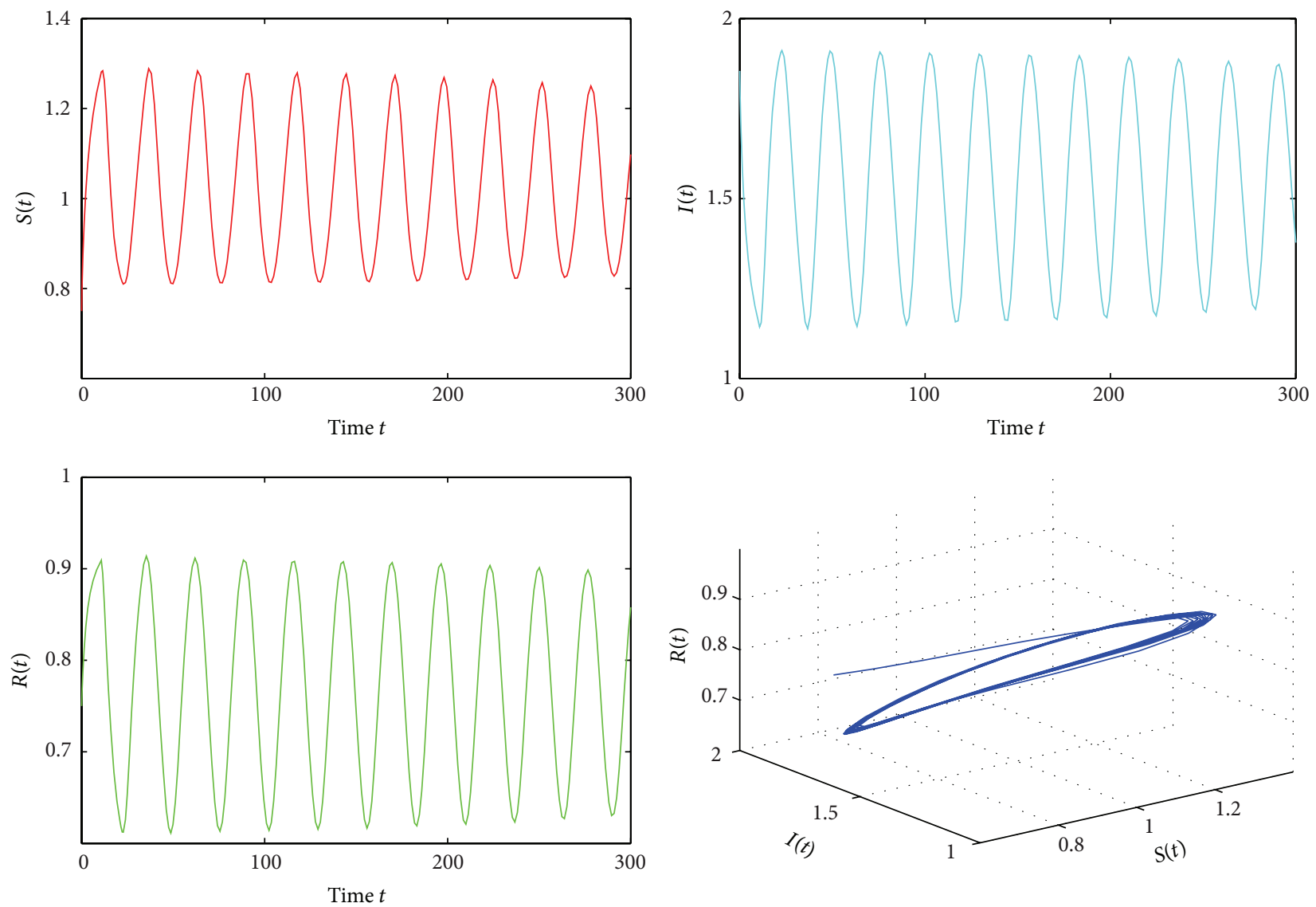

FIgURE 4: $E_{*}$ is unstable for $\tau_{2}=10.7500>\tau_{20}=8.4669$.

By the Riesz representation theorem, there exists a function $\eta(\theta, \mu)$ of bounded variation for $\theta \in[-1,0]$ such that

$$
L_{\mu} \phi=\int_{-1}^{0} d \eta(\theta, \mu) \phi(\theta), \quad \phi \in C\left([-1,0], R^{3}\right) .
$$

In fact, we can choose

$$
\eta(\theta, \mu)= \begin{cases}\left(\tau_{20}^{*}+\mu\right)\left(A^{\prime}+B^{\prime}+C^{\prime}\right), & \theta=0, \\ \left(\tau_{20}^{*}+\mu\right)\left(B^{\prime}+C^{\prime}\right), & \theta \in\left[-\frac{\tau_{1 *}}{\tau_{20}^{*}}, 0\right), \\ \left(\tau_{20}^{*}+\mu\right) C^{\prime}, & \theta \in\left(-1,-\frac{\tau_{1 *}}{\tau_{20}^{*}}\right), \\ 0, & \theta=-1 .\end{cases}
$$

For $\phi \in C\left([-1,0], R^{3}\right)$, we define

$$
\begin{gathered}
A(\mu) \phi= \begin{cases}\frac{d \phi(\theta)}{d \theta}, & -1 \leq \theta<0, \\
\int_{-1}^{0} d \eta(\theta, \mu) \phi(\theta), & \theta=0,\end{cases} \\
R(\mu) \phi= \begin{cases}0, & -1 \leq \theta<0, \\
F(\mu, \phi), & \theta=0 .\end{cases}
\end{gathered}
$$

Then, system (54) is equivalent to

$$
\dot{u}(t)=A(\mu) u_{t}+R(\mu) u_{t},
$$

where $u_{t}=u(t+\theta)$ for $\theta \in[-1,0]$.

For $\varphi \in C\left([-1,0],\left(R^{3}\right)^{*}\right)$, define

$$
A^{*}(\varphi)= \begin{cases}-\frac{d \varphi(s)}{d s}, & 0<s \leq 1, \\ \int_{-1}^{0} d \eta^{T}(s, 0) \varphi(-s), & s=0,\end{cases}
$$

and the bilinear form

$$
\begin{aligned}
& \langle\varphi(s), \phi(\theta)\rangle \\
& \quad=\bar{\varphi}(0) \phi(0)-\int_{\theta=-1}^{0} \int_{\xi=0}^{\theta} \bar{\varphi}(\xi-\theta) d \eta(\theta) \phi(\xi) d \xi,
\end{aligned}
$$

where $\eta(\theta)=\eta(\theta, 0)$.

Let $q(\theta)=\left(1, q_{2}, q_{3}\right)^{T} e^{i \omega_{20}^{*} \tau_{20}^{*} \theta}$ and $q^{*}(s)=V\left(1, q_{2}^{*}\right.$, $\left.q_{3}^{*}\right) e^{i \omega_{20}^{*} \tau_{20}^{*} s}$ be the eigenvectors of $A$ and $A^{*}$ corresponding to 

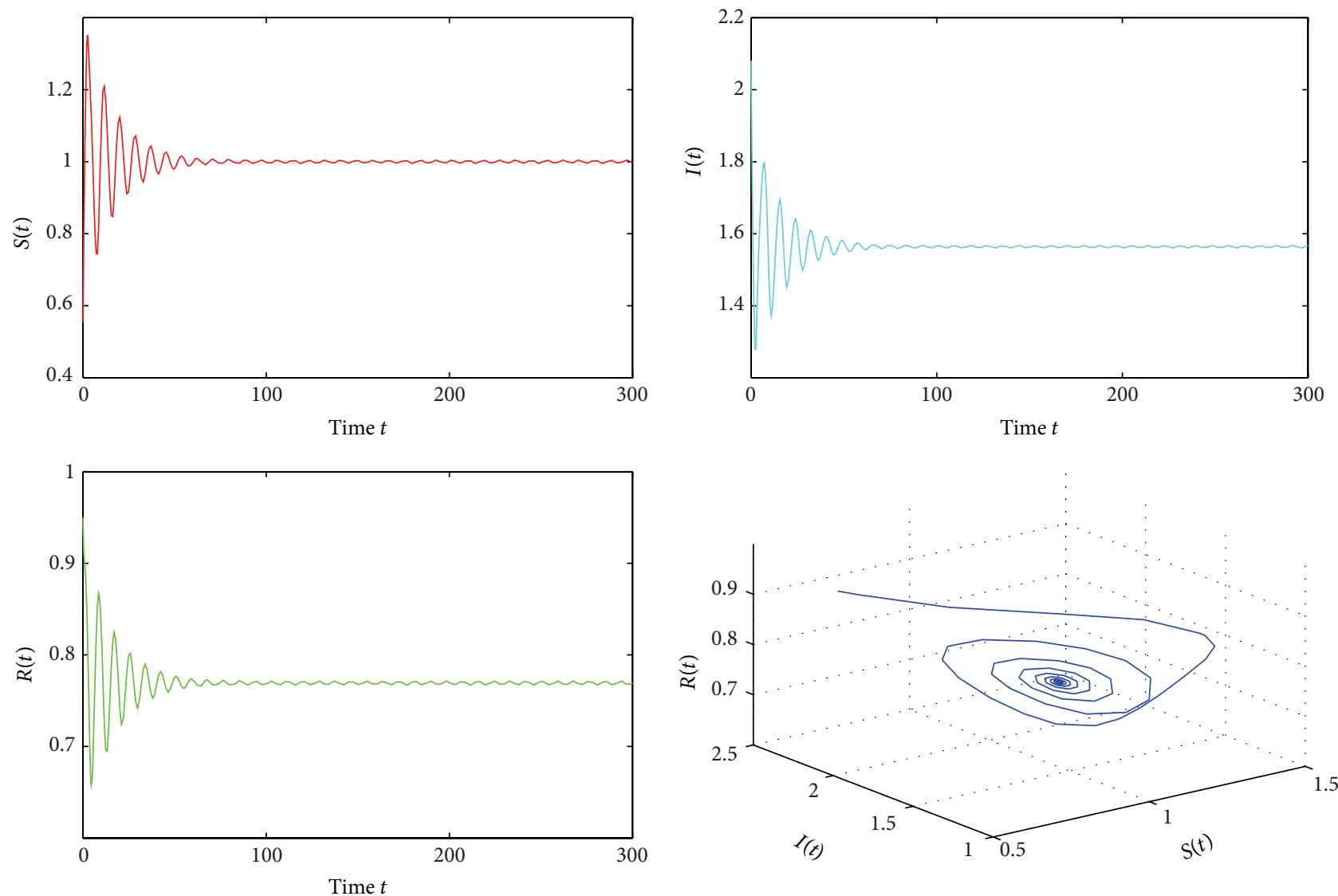

FIGURE 5: $E_{*}$ is locally asymptotically stable for $\tau_{1}=2.0300<\tau_{10}^{*}=2.5386$ and $\tau_{2}=1.05$.

$+i \omega_{20}^{*} \tau_{20}^{*}$ and $-i \omega_{20}^{*} \tau_{20}^{*}$, respectively. By a direction computation, we get

$$
\begin{gathered}
q_{2}=\frac{b_{21} e^{-i \omega_{20}^{*} \tau_{1 *}}}{i \omega_{20}^{*}-a_{22}-b_{22} e^{-i \omega_{20}^{*} \tau_{1 *}}-c_{22} e^{-i \omega_{20}^{*} \tau_{20}^{*}}}, \\
q_{3}=\frac{i \omega_{20}^{*}-a_{11}-b_{11} e^{-i \omega_{20}^{*} \tau_{1 *}}-b_{12} e^{-i \omega_{20}^{*} \tau_{1 *}} q_{2}}{b_{13} e^{-i \omega_{20}^{*} \tau_{1 *}}}, \\
q_{2}^{*}=-\frac{b_{12} e^{-i \omega_{20}^{*} \tau_{1 *}}+c_{32} e^{-i \omega_{20}^{*} \tau_{20}^{*}} q_{3}^{*}}{i \omega_{20}^{*}+a_{22}+b_{22} e^{-i \omega_{20}^{*} \tau_{1 *}}+c_{22} e^{-i \omega_{20}^{*} \tau_{20}^{*}}}, \\
q_{3}^{*}=-\frac{b_{13} e^{-i \omega_{20}^{*} \tau_{1 *}}}{i \omega_{20}^{*}+a_{33}+b_{33} e^{-i \omega_{20}^{*} \tau_{1 *}}} .
\end{gathered}
$$

From (62), we obtain

$$
\begin{gathered}
\bar{V}=\left[1+q_{2} \bar{q}_{2}^{*}+q_{3} \bar{q}_{3}^{*}+\tau_{20}^{*} e^{-i \omega_{20}^{*} \tau_{20}^{*}} q_{2}\left(c_{22} \bar{q}_{2}^{*}+c_{32} \bar{q}_{3}^{*}\right)\right. \\
+\tau_{1 *} e^{-i \omega_{20}^{*} \tau_{1 *}}\left(b_{11}+b_{12} q_{2}+b_{13} q_{3}\right. \\
\left.\left.+\bar{q}_{2}^{*}\left(b_{21}+b_{22} q_{2}\right)+b_{33} q_{3} \bar{q}_{3}^{*}\right)\right]^{-1} .
\end{gathered}
$$

Then, one can see that $\left\langle q^{*}, q\right\rangle=1$ and $\left\langle q^{*}, \bar{q}\right\rangle=0$.
Next, we can obtain the coefficients determining the properties of the Hopf bifurcation by the algorithms introduced in [17] and using a computation process similar to that in $[19,20]$ :

$$
\begin{gathered}
g_{20}=2 \beta \tau_{20}^{*} \bar{V}\left(\bar{q}_{2}^{*}-1\right) q^{(1)}\left(-\frac{\tau_{1 *}}{\tau_{20}^{*}}\right) q^{(2)}\left(-\frac{\tau_{1 *}}{\tau_{20}^{*}}\right) \\
g_{11}=\beta \tau_{20}^{*} \bar{V}\left(\bar{q}_{2}^{*}-1\right)\left[q^{(1)}\left(-\frac{\tau_{1 *}}{\tau_{20}^{*}}\right) \bar{q}^{(2)}\left(-\frac{\tau_{1 *}}{\tau_{20}^{*}}\right)\right. \\
\left.+\bar{q}^{(1)}\left(-\frac{\tau_{1 *}}{\tau_{20}^{*}}\right) q^{(2)}\left(-\frac{\tau_{1 *}}{\tau_{20}^{*}}\right)\right], \\
g_{02}=2 \beta \tau_{20}^{*} \bar{V}\left(\bar{q}_{2}^{*}-1\right) \bar{q}^{(1)}\left(-\frac{\tau_{1 *}}{\tau_{20}^{*}}\right) \bar{q}^{(2)}\left(-\frac{\tau_{1 *}}{\tau_{20}^{*}}\right) \\
g_{21}=2 \beta \tau_{20}^{*} \bar{V}\left(\bar{q}_{2}^{*}-1\right)\left[W_{11}^{(1)}\left(-\frac{\tau_{1 *}}{\tau_{20}^{*}}\right) q^{(2)}\left(-\frac{\tau_{1 *}}{\tau_{20}^{*}}\right)\right. \\
+\frac{1}{2} W_{20}^{(1)}\left(-\frac{\tau_{1 *}}{\tau_{20}^{*}}\right) \bar{q}^{(2)}\left(-\frac{\tau_{1 *}}{\tau_{20}^{*}}\right)
\end{gathered}
$$



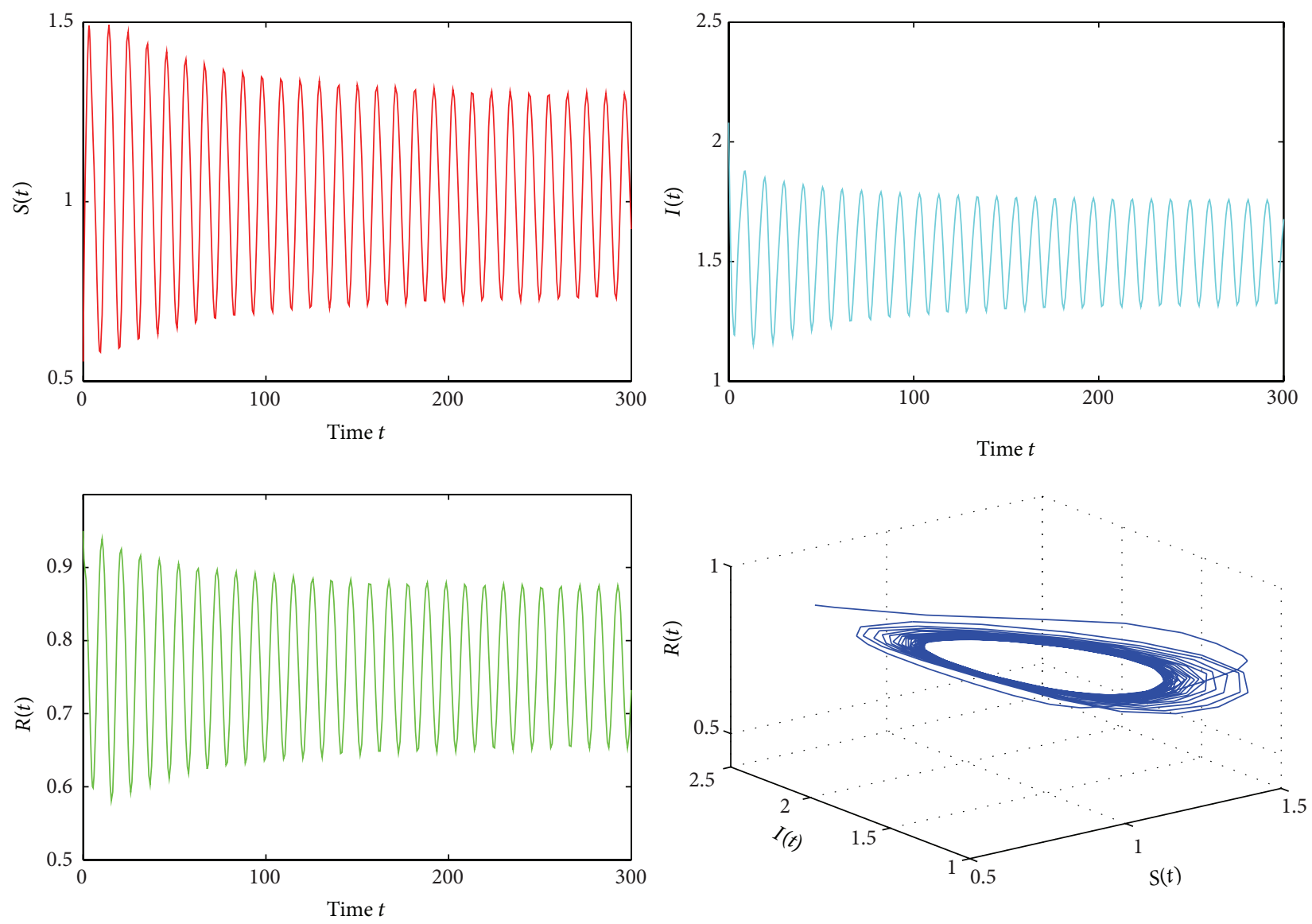

FIGURE 6: $E_{*}$ is unstable for $\tau_{1}=2.9732>\tau_{10}^{*}=2.5386$ and $\tau_{2}=1.05$.

$$
\begin{aligned}
& +W_{11}^{(2)}\left(-\frac{\tau_{1 *}}{\tau_{20}^{*}}\right) q^{(1)}\left(-\frac{\tau_{1 *}}{\tau_{20}^{*}}\right) \\
& \left.+\frac{1}{2} W_{20}^{(2)}\left(-\frac{\tau_{1 *}}{\tau_{20}^{*}}\right) \bar{q}^{(1)}\left(-\frac{\tau_{1 *}}{\tau_{20}^{*}}\right)\right],
\end{aligned}
$$

$$
\begin{gathered}
\left(\begin{array}{ccc}
a_{11}+b_{11} & b_{12} & b_{13} \\
b_{21} & a_{22}+b_{22}+c_{22} & 0 \\
a_{31} & c_{32} & a_{33}+b_{33}
\end{array}\right) E_{11} \\
=-\left(\begin{array}{c}
E_{11}^{(1)} \\
E_{11}^{(2)} \\
0
\end{array}\right)
\end{gathered}
$$

with

$$
\begin{gathered}
W_{20}(\theta)=\frac{i g_{20} q(0)}{\omega_{20}^{*} \tau_{20}^{*}} e^{i \omega_{20}^{*} \tau_{20}^{*} \theta}+\frac{i \bar{g}_{02} \bar{q}(0)}{3 \omega_{20}^{*} \tau_{20}^{*}} e^{-i \omega_{20}^{*} \tau_{20}^{*} \theta} \\
+E_{20} e^{2 i \omega_{20}^{*} \tau_{20}^{*} \theta}, \\
W_{11}(\theta)=-\frac{i g_{11} q(0)}{\omega_{20}^{*} \tau_{20}^{*}} e^{i \omega_{20}^{*} \tau_{20}^{*} \theta}+\frac{i \bar{g}_{11} \bar{q}(0)}{\omega_{20}^{*} \tau_{20}^{*}} e^{-i \omega_{20}^{*} \tau_{20}^{*} \theta}+E_{11},
\end{gathered}
$$

where $E_{20}$ and $E_{11}$ can be calculated by the following two equations:

$$
\begin{aligned}
& \left(\begin{array}{ccc}
a_{11}^{\prime} & -b_{12} e^{-2 i \omega_{20}^{*} \tau_{1 *}} & -b_{13} e^{-2 i \omega_{20}^{*} \tau_{1 *}} \\
-b_{21} e^{-2 i \omega_{20}^{*} \tau_{1 *}} & a_{22}^{\prime} & 0 \\
-a_{31} & -c_{32} e^{-2 i \omega_{20}^{*} \tau_{20}^{*}} & a_{33}^{\prime}
\end{array}\right) E_{20} \\
& =2\left(\begin{array}{c}
E_{20}^{(1)} \\
E_{20}^{(2)} \\
0
\end{array}\right),
\end{aligned}
$$

with

$$
\begin{aligned}
& a_{11}^{\prime}= 2 i \omega_{20}^{*}-a_{11}-b_{11} e^{-2 i \omega_{20}^{*} \tau_{1 *}}, \\
& a_{22}^{\prime}= 2 i \omega_{20}^{*}-a_{22}-b_{22} e^{-2 i \omega_{20}^{*} \tau_{1 *}}-c_{22} e^{-2 i \omega_{20}^{*} \tau_{20}^{*}}, \\
& a_{33}^{\prime}= 2 i \omega_{20}^{*}-a_{33}-b_{33} e^{-2 i \omega_{20}^{*} \tau_{1 *}}, \\
& E_{20}^{(1)}=-\beta q^{(1)}\left(-\frac{\tau_{1 *}}{\tau_{20}^{*}}\right) q^{(2)}\left(-\frac{\tau_{1 *}}{\tau_{20}^{*}}\right), \\
& E_{20}^{(2)}=\beta q^{(1)}\left(-\frac{\tau_{1 *}}{\tau_{20}^{*}}\right) q^{(2)}\left(-\frac{\tau_{1 *}}{\tau_{20}^{*}}\right) \\
& E_{11}^{(1)}=-\beta\left[q^{(1)}\left(-\frac{\tau_{1 *}}{\tau_{20}^{*}}\right) \bar{q}^{(2)}\left(-\frac{\tau_{1 *}}{\tau_{20}^{*}}\right)\right. \\
&\left.\quad+\bar{q}^{(1)}\left(-\frac{\tau_{1 *}}{\tau_{20}^{*}}\right) q^{(2)}\left(-\frac{\tau_{1 *}}{\tau_{20}^{*}}\right)\right],
\end{aligned}
$$



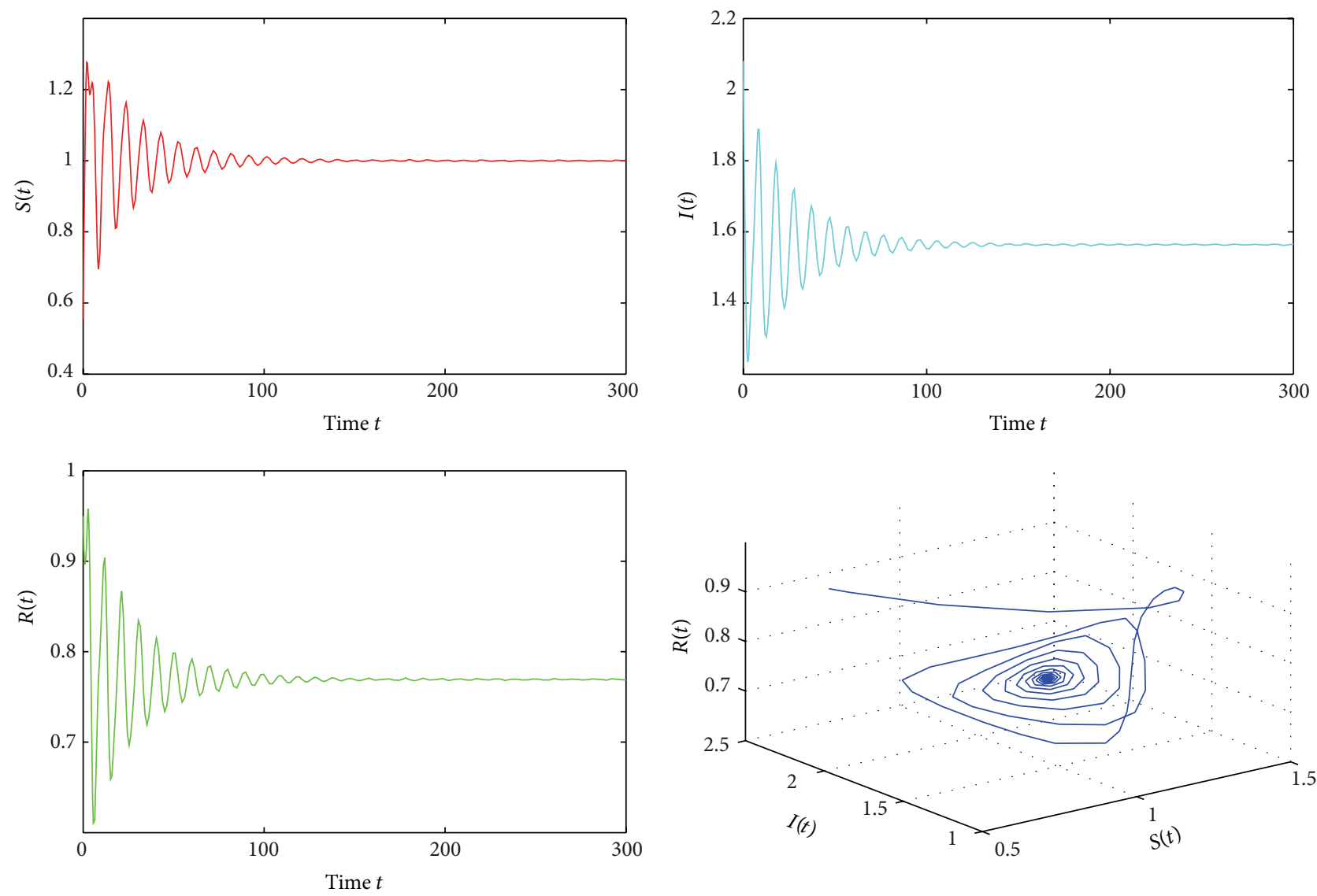

FIGURE 7: $E_{*}$ is locally asymptotically stable for $\tau_{2}=2.3700<\tau_{20}^{*}=2.9170$ and $\tau_{1}=1.65$.

$$
\begin{aligned}
E_{11}^{(2)}=\beta\left[q^{(1)}\left(-\frac{\tau_{1 *}}{\tau_{20}^{*}}\right) \bar{q}^{(2)}\left(-\frac{\tau_{1 *}}{\tau_{20}^{*}}\right)\right. \\
\left.+\bar{q}^{(1)}\left(-\frac{\tau_{1 *}}{\tau_{20}^{*}}\right) q^{(2)}\left(-\frac{\tau_{1 *}}{\tau_{20}^{*}}\right)\right] .
\end{aligned}
$$

Then, we can get the following coefficients:

$$
\begin{gathered}
C_{1}(0)=\frac{i}{2 \omega_{20}^{*} \tau_{20}^{*}}\left(g_{11} g_{20}-2\left|g_{11}\right|^{2}-\frac{\left|g_{02}\right|^{2}}{3}\right)+\frac{g_{21}}{2}, \\
\mu_{2}=-\frac{\operatorname{Re}\left\{C_{1}(0)\right\}}{\operatorname{Re}\left\{\lambda^{\prime}\left(\tau_{20}^{*}\right)\right\}}, \\
\beta_{2}=2 \operatorname{Re}\left\{C_{1}(0)\right\}, \\
T_{2}=-\frac{\operatorname{Im}\left\{C_{1}(0)\right\}+\mu_{2} \operatorname{Im}\left\{\lambda^{\prime}\left(\tau_{20}^{*}\right)\right\}}{\omega_{20}^{*} \tau_{20}^{*}} .
\end{gathered}
$$

By the discussion above, we have the following results about the properties of the Hopf bifurcation.

Theorem 6. For system (3), the direction of the Hopf bifurcation is determined by the sign of $\mu_{2}$ : if $\mu_{2}>0\left(\mu_{2}<0\right)$, the Hopf bifurcation is supercritical (subcritical); the stability of

bifurcating periodic solutions is determined by the sign of $\beta_{2}$ : if $\beta_{2}<0\left(\beta_{2}>0\right)$, the bifurcating periodic solutions are stable (unstable); the period of the bifurcating periodic solutions is determined by the sign of $T_{2}$ : if $T_{2}>0\left(T_{2}<0\right)$, the period of the bifurcating periodic solutions increases (decreases).

\section{Numerical Simulations and Discussion}

In this section, in order to support our theoretical results, we will show the interesting dynamical behaviors of system (3) by a special case of system (3). Let $p=0.9, A=1$, let $\alpha=0.3$, $\beta=0.6, \gamma=0.2, \delta=0.7$, and $\mu=0.3$ and we consider the following system:

$$
\begin{aligned}
& \frac{d S(t)}{d t}=0.9-0.6 S\left(t-\tau_{1}\right) I\left(t-\tau_{1}\right)-0.5 S(t) \\
& +0.7 R\left(t-\tau_{1}\right) \\
& \frac{d I(t)}{d t}=0.6 S\left(t-\tau_{1}\right) I\left(t-\tau_{1}\right)-0.3 I(t)-0.3 I\left(t-\tau_{2}\right), \\
& \frac{d R(t)}{d t}=0.1+0.2 S(t)+0.3 I\left(t-\tau_{2}\right)-0.7 R\left(t-\tau_{1}\right) \\
& -0.3 R(t)
\end{aligned}
$$



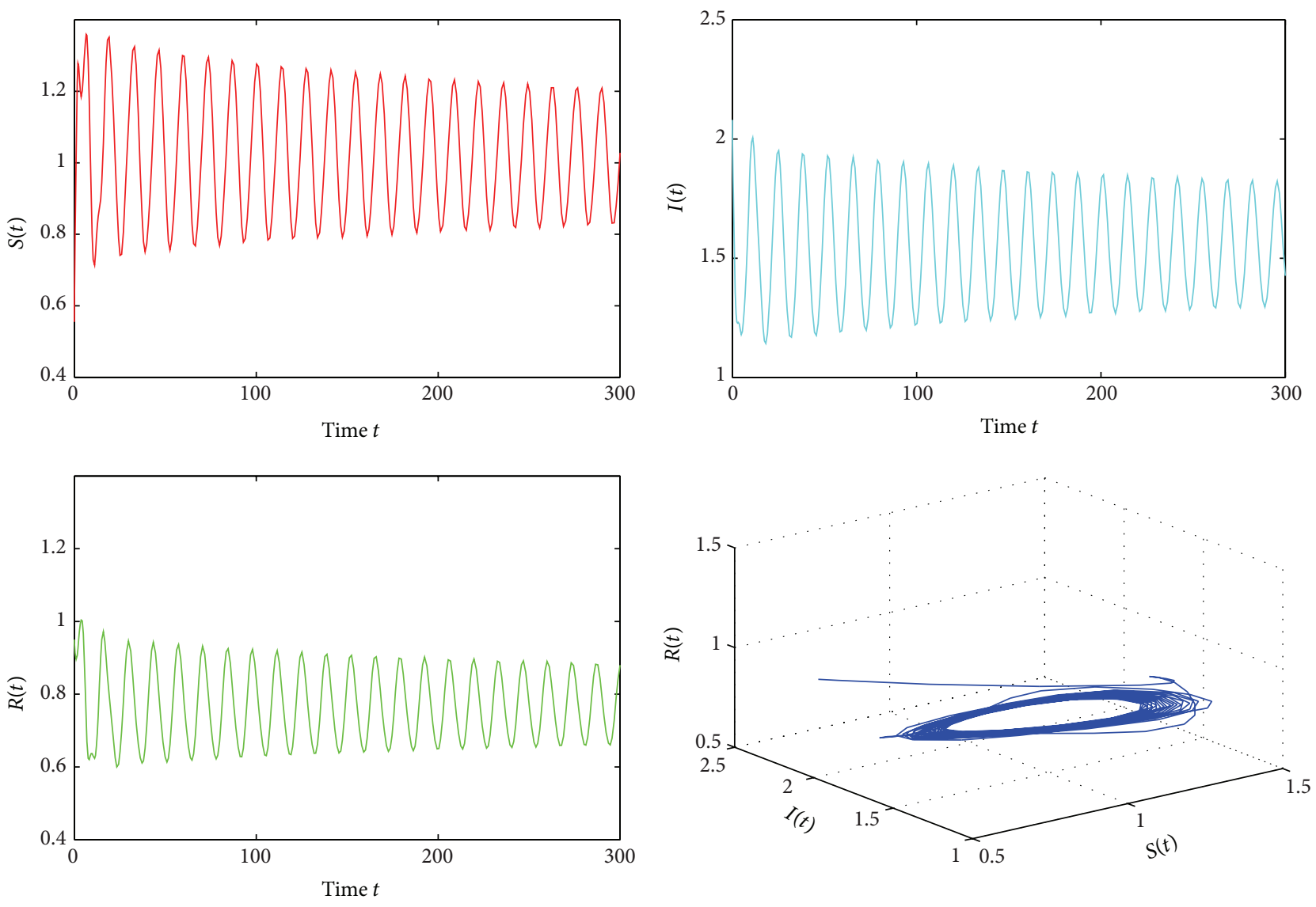

FIGURE 8: $E_{*}$ is unstable for $\tau_{2}=4.3500>\tau_{20}^{*}=2.9170$ and $\tau_{1}=1.65$.

from which one can get $R_{0}=2.2200$ and the unique positive equilibrium $E_{*}(1.0000,1.5641,0.7692)$. By computing, we obtain $A_{10}=0.3661, A_{11}=1.8616$, and $A_{12}=2.4385$. Obviously, $A_{12} A_{11}>A_{10}>0$.

For $\tau_{1}>0, \tau_{2}=0$. Equation (18) has a unique positive root $\omega_{10}=0.5833$ and one can obtain $\tau_{10}=2.8957$ from (21). Further, the characteristic equation (14) has a pair of purely imaginary roots $\pm i \omega_{10}$. The computer simulations in Figures 1 and 2 show that $E_{*}(1.0000,1.5641,0.7692)$ is asymptotically stable when $\tau_{1}<\tau_{10}=2.8957$ and when $\tau_{1}$ passes through the critical value $\tau_{10}=2.8957, E_{*}(1.0000,1.5641,0.7692)$ loses its stability and a Hopf bifurcation occurs; that is, a family of periodic solutions bifurcate from $E_{*}(1.0000,1.5641,0.7692)$. Similarly, we obtain $\omega_{20}=3.2514$ and $\tau_{20}=8.4669$. The corresponding trajectories graphs and phase graphs are shown in Figures 3 and 4 .

Let $\tau_{2}=1.05 \in\left(0, \tau_{20}\right)$ and choose $\tau_{1}$ as a bifurcation parameter. Then, we have $\omega_{10}^{*}=1.0514$ and $\tau_{10}^{*}=2.5386$. The computer simulations in Figures 5 and 6 show that $E_{*}(1.0000,1.5641,0.7692)$ is asymptotically stable when $\tau_{1}<$ $\tau_{10}^{*}=2.5386$ and $E_{*}(1.0000,1.5641,0.7692)$ loses its stability and a Hopf bifurcation occurs; that is, a family of periodic solutions bifurcate from $E_{*}(1.0000,1.5641,0.7692)$. Similarly, by some complex computations, we have $\omega_{20}^{*}=0.8545$ and $\tau_{20}^{*}=2.9170$ when $\tau_{1}=1.65 \in\left(0, \tau_{10}\right)$ and choose $\tau_{2}$ as a bifurcation parameter. The corresponding trajectories graphs and phase graphs are shown in Figures 7 and 8. Furthermore, we can compute and obtain $\lambda^{\prime}\left(\tau_{20}^{*}\right)=2.3606+1.7054 i$ and $C_{1}(0)=-17.9318+26.0921 i$. It follows from (69) that $\mu_{2}=7.5963>0, \beta_{2}=-35.8636<0$, and $T_{2}=-15.3981<0$. According to Theorem 6, we can conclude that the Hopf bifurcation of system (70) is supercritical, the bifurcating periodic solutions are stable, and the period of the periodic solutions decreases.

In addition, it can be seen from the expression of the positive equilibrium of system (3) that the more the hosts are attached to the computer networks, the more the hosts in networks will be infected. Therefore, the managers of the real networks should properly control the number of the new hosts attached to networks. According to the numerical simulations, we also find that the onset of the Hopf bifurcation can be delayed by the values of the parameters $A$ and $p$ in system (3), which can be controlled by the managers of the real networks. Therefore, the managers of the real networks should properly control the number of the hosts attached to the networks and properly strengthen the immunization of the new hosts in order to control the onset of the Hopf bifurcation, so as to make the propagation of computer viruses predicted and controlled easily. 


\section{Conclusion}

In this paper, an SIRS computer virus propagation model with two delays and multistate antivirus measures is investigated. By choosing the possible combination of the two delays as the bifurcation parameter and analyzing the distribution of the roots of the associated characteristic equation, sufficient conditions for the local stability of the positive equilibrium and existence of local Hopf bifurcation are obtained. Furthermore, the properties of the Hopf bifurcation are determined by using the method in [17].

Compared to the model considered in [12], we consider not only the delay due to the latent period of computer virus and the delay due to the temporary immune period of the recovered hosts, but also the delay due to the period that the antivirus software uses to clean the viruses in the infected hosts. All the possible delays are incorporated into the model and the model considered in this paper is more general. Our analysis shows that the new delay we incorporate into the model can also change the stability of the positive equilibrium of the model and numerical simulations show that our results obtained in the present paper improve some of the existing results on this system that are obtained in [12].

\section{Conflict of Interests}

The authors declare that there is no conflict of interests regarding the publication of this paper.

\section{Acknowledgments}

The authors are grateful to the anonymous referees and the editor for their valuable comments and suggestions on the paper. This work was supported by the National Natural Science Foundation of China (61273070), a project funded by the Priority Academic Program Development of Jiangsu Higher Education Institutions, and Natural Science Foundation of the Higher Education Institutions of Anhui Province (KJ2014A005).

\section{References}

[1] W. H. Murray, "The application of epidemiology to computer viruses," Computers and Security, vol. 7, no. 2, pp. 139-150, 1988.

[2] J. O. Kephart and S. R. White, "Directed-graph epidemiological models of computer viruses," in Proceedings of the IEEE Computer Society Symposium on Research in Security and Privacy, pp. 343-359, May 1991.

[3] J. O. Kephart and S. R. White, "Measuring and modeling computer virus prevalence," in Proceedings of the IEEE Computer Society Symposium on Research in Security and Privacy, pp. 2-15, May 1993.

[4] C. C. Zou, D. Towsley, and W. Gong, "On the performance of Internet worm scanning strategies," Performance Evaluation, vol. 63 , no. 7, pp. 700-723, 2006.

[5] H. Yuan and G. Chen, "Network virus-epidemic model with the point-to-group information propagation," Applied Mathematics and Computation, vol. 206, no. 1, pp. 357-367, 2008.
[6] T. Dong, X. Liao, and H. Li, "Stability and Hopf bifurcation in a computer virus model with multistate antivirus," Abstract and Applied Analysis, vol. 2012, Article ID 841987, 16 pages, 2012.

[7] B. K. Mishra and S. K. Pandey, "Dynamic model of worms with vertical transmission in computer network," Applied Mathematics and Computation, vol. 217, no. 21, pp. 8438-8446, 2011.

[8] B. K. Mishra and N. Jha, "SEIQRS model for the transmission of malicious objects in computer network," Applied Mathematical Modelling, vol. 34, no. 3, pp. 710-715, 2010.

[9] F. Wang, Y. Zhang, C. Wang, J. Ma, and S. Moon, "Stability analysis of a SEIQV epidemic model for rapid spreading worms," Computers \& Security, vol. 29, no. 4, pp. 410-418, 2010.

[10] B. K. Mishra and N. Keshri, "Mathematical model on the transmission of worms in wireless sensor network," Applied Mathematical Modelling, vol. 37, no. 6, pp. 4103-4111, 2013.

[11] J. Ren, X. Yang, L.-X. Yang, Y. Xu, and F. Yang, "A delayed computer virus propagation model and its dynamics," Chaos, Solitons \& Fractals, vol. 45, no. 1, pp. 74-79, 2012.

[12] L. Feng, X. Liao, H. Li, and Q. Han, "Hopf bifurcation analysis of a delayed viral infection model in computer networks," Mathematical and Computer Modelling, vol. 56, no. 7-8, pp. 167179, 2012.

[13] B. K. Mishra and D. K. Saini, "SEIRS epidemic model with delay for transmission of malicious objects in computer network," Applied Mathematics and Computation, vol. 188, no. 2, pp. 14761482, 2007.

[14] Q. Y. Zhu, X. F. Liao, L. X. Yang, and C. M. Zhang, "Optimal control of computer virus under a delayed model," Applied Mathematics and Computation, vol. 218, no. 23, pp. 11613-11619, 2012.

[15] J. Liu, "Hopf bifurcation in a delayed SEIQRS model for the transmission of malicious objects in computer network," Journal of Applied Mathematics, vol. 2014, Article ID 492198, 8 pages, 2014.

[16] W. O. Kermack and A. G. McKendrick, "Contributions to the mathematical theory of pidemics," Proceedings of the Royal Society of London Series A, vol. 115, no. 772, pp. 700-721, 1927.

[17] B. D. Hassard, N. D. Kazarinoff, and Y. H. Wan, Theory and Applications of Hopf Bifurcation, Cambridge University Press, Cambridge, UK, 1981.

[18] Y. Song, M. Han, and J. Wei, "Stability and Hopf bifurcation analysis on a simplified BAM neural network with delays," Physica D: Nonlinear Phenomena, vol. 200, no. 3-4, pp. 185-204, 2005.

[19] C. Xu and X. He, "Stability and bifurcation analysis in a class of two-neuron networks with resonant bilinear terms," Abstract and Applied Analysis, vol. 2011, Article ID 697630, 21 pages, 2011.

[20] C. Bianca, M. Ferrara, and L. Guerrini, "The Cai model with time delay: existence of periodic solutions and asymptotic analysis," Applied Mathematics and Information and Sciences, vol. 7, no. 1, pp. 21-27, 2013. 


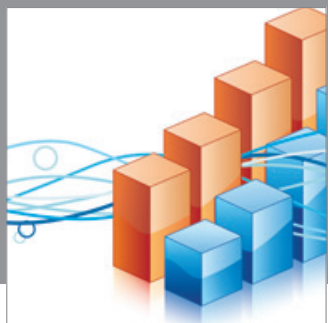

Advances in

Operations Research

mansans

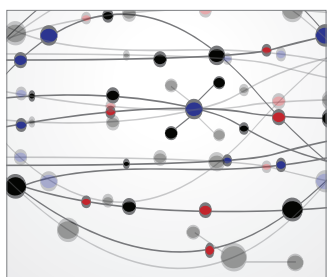

The Scientific World Journal
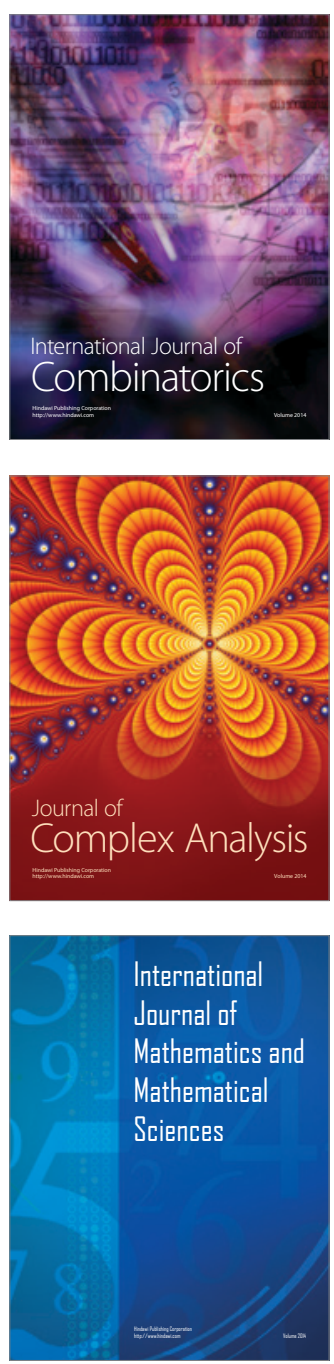
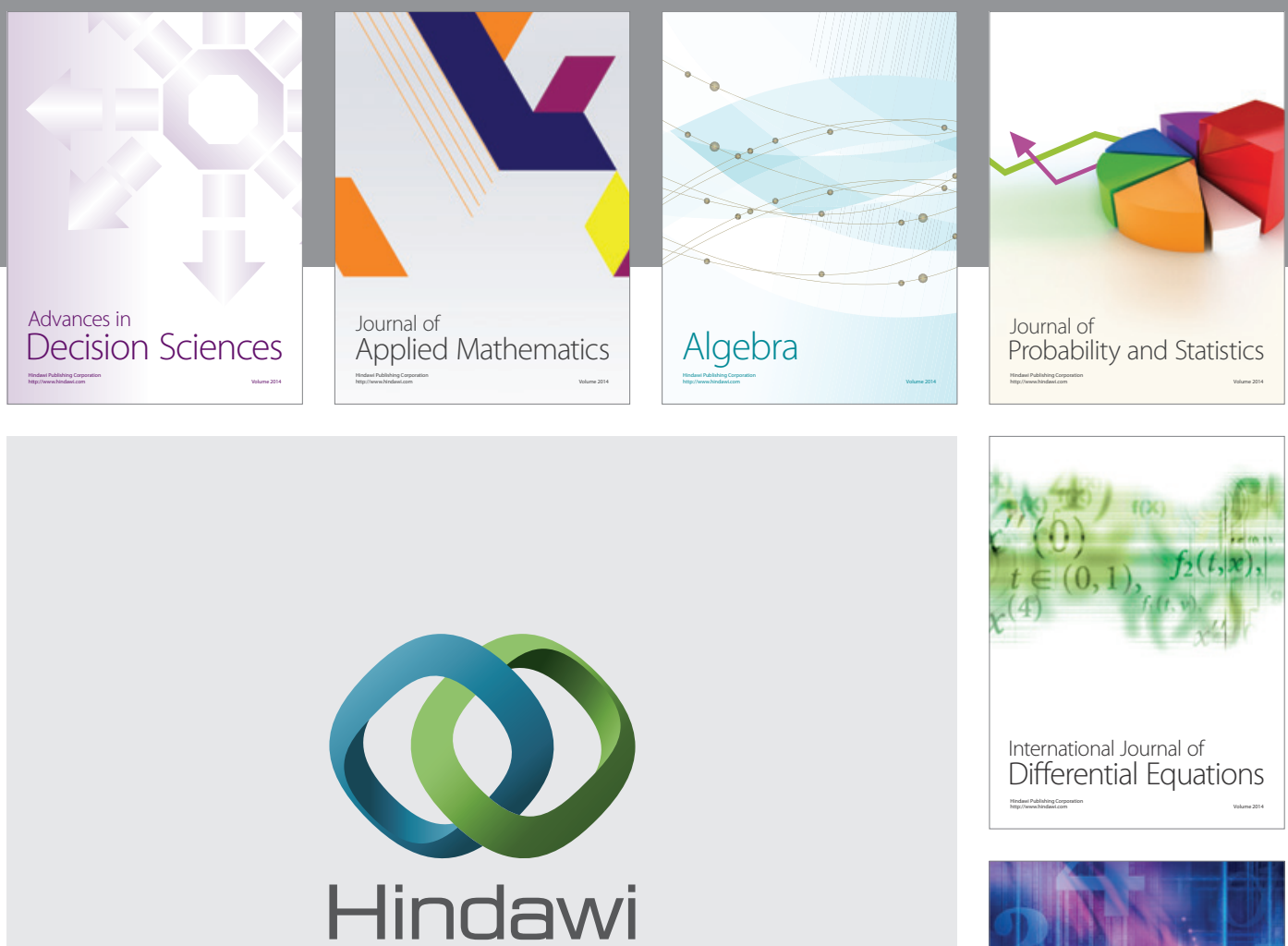

Submit your manuscripts at http://www.hindawi.com
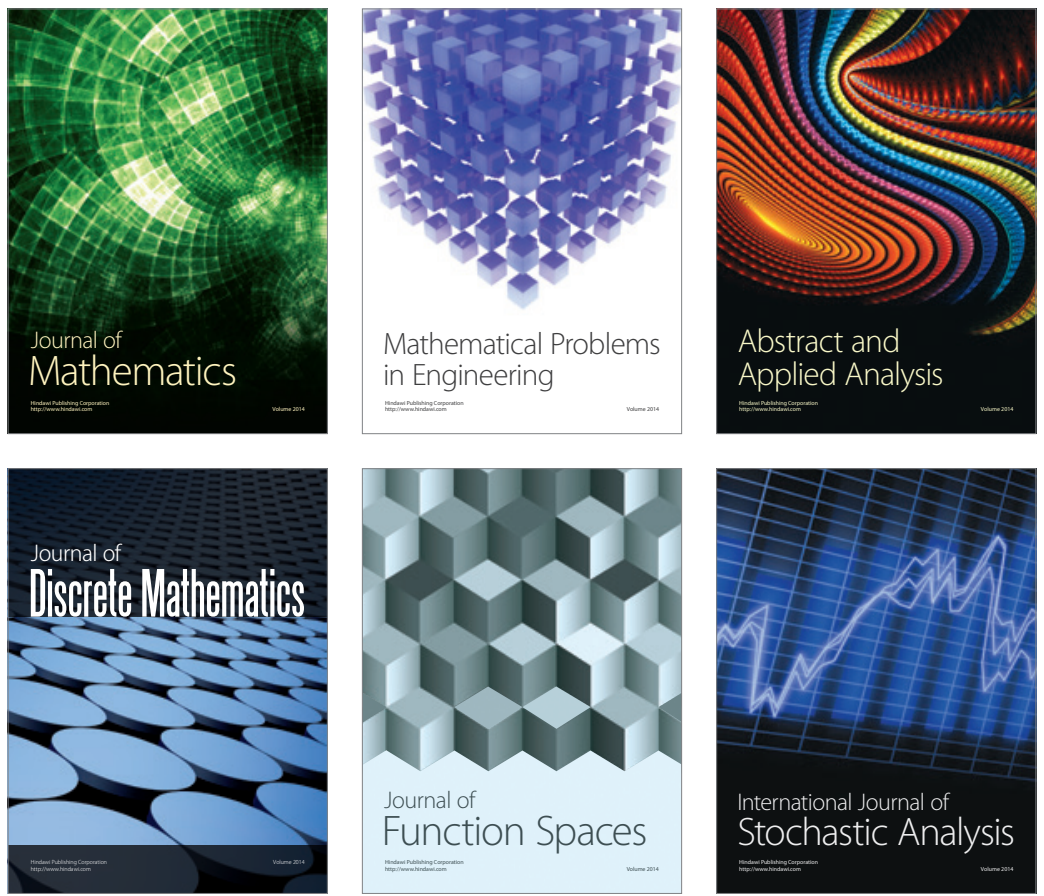

Journal of

Function Spaces

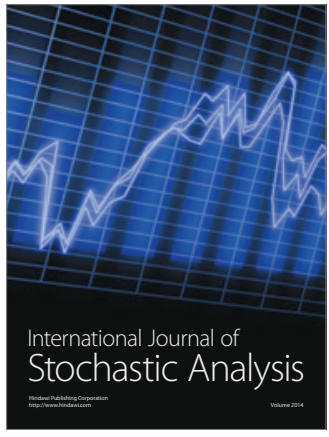

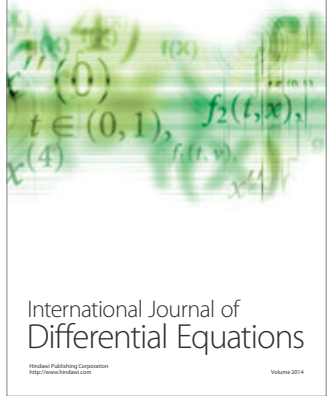
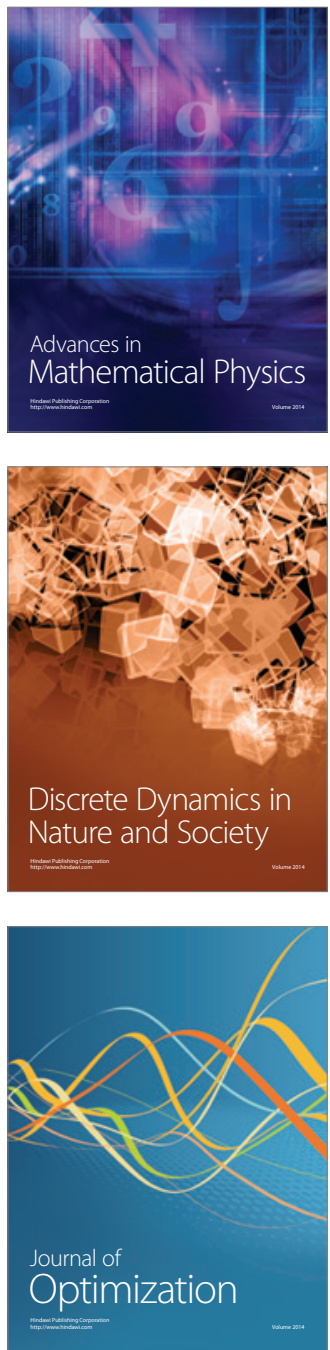